\title{
A PUF Hub Drives Self-Renewal in Caenorhabditis elegans Germline Stem Cells
}

\author{
Kimberly A. Haupt, Kimberley T. Law, ${ }^{1}$ Amy L. Enright, Charlotte R. Kanzler, Heaji Shin, ${ }^{2}$ \\ Marvin Wickens, and Judith Kimble ${ }^{3}$ \\ Department of Biochemistry, University of Wisconsin-Madison, Wisconsin 53706 \\ ORCID IDs: 0000-0003-1109-3129 (K.A.H.); 0000-0001-5208-5999 (H.S.); 0000-0002-0593-5740 (M.W.); 0000-0001-5622-2073 (J.K.)
}

\begin{abstract}
Stem cell regulation relies on extrinsic signaling from a niche plus intrinsic factors that respond and drive self-renewal within stem cells. A priori, loss of niche signaling and loss of the intrinsic self-renewal factors might be expected to have equivalent stem cell defects. Yet this simple prediction has not been borne out for most stem cells, including Caenorhabditis elegans germline stem cells (GSCS). The central regulators of $C$. elegans GSCs include extrinsically acting GLP-1/Notch signaling from the niche; intrinsically acting RNA-binding proteins in the PUF family, termed FBF-1 and FBF-2 (collectively FBF); and intrinsically acting PUF partner proteins that are direct Notch targets. Abrogation of either GLP-1/Notch signaling or its targets yields an earlier and more severe GSC defect than loss of FBF-1 and FBF-2, suggesting that additional intrinsic regulators must exist. Here, we report that those missing regulators are two additional PUF proteins, PUF-3 and PUF-11. Remarkably, an fbf-1 fbf-2; puf-3 puf-11 quadruple null mutant has a GSC defect virtually identical to that of a g/p-1/Notch null mutant. PUF-3 and PUF-11 both affect GSC maintenance, both are expressed in GSCs, and epistasis experiments place them at the same position as FBF within the network. Therefore, action of PUF-3 and PUF-11 explains the milder GSC defect in fbf-1 fbf-2 mutants. We conclude that a "PUF hub," comprising four PUF proteins and two PUF partners, constitutes the intrinsic self-renewal node of the C. elegans GSC RNA regulatory network. Discovery of this hub underscores the significance of PUF RNA-binding proteins as key regulators of stem cell maintenance.
\end{abstract}

KEYWORDS stem cell regulatory network; redundancy; FBF; PUF-3; PUF-11

TEM cell regulatory networks govern the balance between self-renewal and differentiation. Transcription factors are well-established stem cell regulators (e.g., Boyer et al. 2005), as are RNA-binding proteins (e.g., Wickens et al. 2002; Ye and Blelloch 2014; Gross-Thebing et al. 2017). Indeed, the PUF (for Pumilio and FBF) family of RNA-binding proteins promote stem cell self-renewal in multiple tissues across animal phylogeny from planaria to mammals (Lin and Spradling 1997; Forbes and Lehmann 1998; Crittenden et al. 2002;

Copyright (C) 2020 by the Genetics Society of America

doi: https://doi.org/10.1534/genetics.119.302772

Manuscript received September 27, 2019; accepted for publication November 5, 2019; published Early Online November 18, 2019

Supplemental material available at figshare: https://doi.org/10.25386/genetics. 10250801.

${ }^{1}$ Present address: School of Veterinary Medicine, University of Pennsylvania, Philadelphia, PA 19104

2Present address: The David H. Koch Institute for Integrative Cancer Research, Massachusetts Institute of Technology, Cambridge, MA 02139.

${ }^{3}$ Corresponding author: Department of Biochemistry, University of WisconsinMadison, 433 Babcock Dr., Madison, WI 53706-1544. E-mail: jekimble@wisc. edu
Salvetti et al. 2005; Naudin et al. 2017; Zhang et al. 2017). Genome-wide studies reveal that PUF proteins bind hundreds of RNAs (Hafner et al. 2010; Kershner and Kimble 2010; Prasad et al. 2016; Porter et al. 2019), consistent with a central role in the stem cell regulatory network (Kershner et al. 2013). Yet the full significance of PUF proteins in self-renewal has been unclear. Are PUF proteins the primary stem-cell-intrinsic self-renewal regulators? Or do they play only a supporting role? This question has been difficult to tackle in organisms where only one or two PUF proteins are responsible for many diverse processes, including embryogenesis, germ cell development, and neural activities. Here we address the question in nematodes, where the number of genes encoding PUF proteins has expanded during evolution, yielding functional specialization among family members.

We focus here on the role of PUF proteins in regulating selfrenewal in the Caenorhabditis elegans germline. Germline stem cells (GSCs) expand the germline tissue from two cells at hatching to $\sim 2000$ cells in adults, they replenish the tissue as germ cells are lost to gametogenesis during reproduction 
(Kimble and White 1981; Crittenden et al. 2006), and they regenerate the tissue upon feeding after starvation (Angelo and Van Gilst 2009; Seidel and Kimble 2011). Key regulators of GSC self-renewal include GLP-1/Notch signaling from the niche; its transcriptional targets, lst-1 and sygl-1; and two PUF proteins, FBF-1 and FBF-2 (collectively FBF) (Figure 1A) (Austin and Kimble 1987; Crittenden et al. 2002; Kershner et al. 2014). The lst-1 and sygl-1 genes encode proteins LST-1 and SYGL-1, which each interact physically with FBF-1 and FBF-2 proteins and are therefore PUF "partners" (Shin et al. 2017; Haupt et al. 2019).

While FBF-1 and FBF-2 are crucial in GSC control, they cannot account for all the effects of niche signaling. Removal of either the GLP-1/Notch receptor or both $l s t-1$ and sygl-1 has an early and severe germline proliferation (Glp) defect in both sexes: the two GSCs at hatching divide once or twice and then differentiate prematurely as sperm (Austin and Kimble 1987; Kershner et al. 2014). Moreover, GLP-1/Notch and its targets drive self-renewal throughout larval development and in adults (Austin and Kimble 1987; Kershner et al. 2014). By contrast, GSCs in the $f b f-1 f b f-2$ double mutant are lost to differentiation much later, at the fourth larval stage (L4s) (Crittenden et al. 2002). Indeed, this delayed L4 GSC defect occurs in mutants raised at 15 and $20^{\circ}$, but at $25^{\circ}$, the effect is even further delayed, with GSCs continuing to divide in adults and persisting in an undifferentiated state (Merritt et al. 2008; Shin et al. 2017; this work). We designate the less severe germline phenotype of $f b f-1 f b f-2$ mutants pGlp ("partial Glp") to distinguish it from the more severe Glp GSC defect seen upon loss of niche signaling. The pGlp defect implies that some other self-renewal factor, "gene X" (Figure 1A), must maintain GSCs in larvae at all temperatures and in adults at $25^{\circ}$.

Here, we report that two additional PUF proteins, PUF- 3 and PUF-11, are gene X. When PUF-3 and PUF-11 are removed in an $f b f-1 f b f-2$ mutant, the GSC defect is essentially the same as when niche signaling is lost. Moreover, PUF-3 and PUF-11 promote GSC self-renewal in both larvae and adults, are expressed in the distal germline where GSCs reside, and physically interact with PUF partners LST-1 and SYGL-1. We conclude that a "PUF hub," comprising four PUF proteins and two PUF partners, constitutes the self-renewal node of the $C$. elegans GSC RNA regulatory network. Discovery of this hub underscores the significance of PUF RNA-binding proteins as key regulators of stem cell maintenance.

\section{Materials and Methods}

\section{Nematode strains and maintenance}

C. elegans strains were maintained at $20^{\circ}$, unless specified otherwise, on nematode growth medium (NGM) plates spotted with Escherichia coli OP50 (Brenner 1974). Wild type was N2 Bristol strain. For a complete list of strains used in this study, see Supplemental Material, Table S1. puf-11(gk203683) is from the Million Mutations Project (Thompson et al. 2013). We also used the following balancers: hT2[qIs48] (I;III) (Siegfried and Kimble 2002), mIn1[mIs14 dpy-10(e128)] II (Edgley and Riddle 2001), and $n T 1$ [qIs51] (IV;V) (Edgley et al. 2006).

Because of incompatibility between $\operatorname{mIn} 1$ and $n T 1$ balancers, we generated a "pseudo $(\Psi)$-balancer" to maintain quadruple mutant strains. This $L G$ II $\Psi$-balancer harbors a transgene driving expression of a red fluorescent protein in somatic nuclei, oxTi564 [Peft-3::tdTomato::H2B::unc-54 3'UTR + Cbr-unc-119(+)] (Frøkjær-Jensen et al. 2014), plus a closely linked dpy-10(q1074) deletion. Quadruple mutants were thus maintained as $f b f-1 f b f-2 / \Psi$-balancer $[d p y-10$ (q1074) oxTi564] II; puf-3 puf-11 IV/nT1[qIs51] (IV;V).

\section{CRISPR/Cas9 genome editing}

We used RNA-protein complex CRISPR/Cas9 genome editing with a coconversion strategy (Arribere et al. 2014; Paix et al. 2015) to generate a number of alleles for this work (see Table S2 for details). For each edit, we prepared an injection mix containing: gene-specific CRISPR RNAs (10 $\mu \mathrm{M}$, IDT-Alt-R); dpy-10 or unc-58 co-CRISPR crRNAs ( $4 \mu \mathrm{M}$, IDT-Alt-R); trans-activating CRISPR RNAs (13.6 $\mu \mathrm{M}$, IDT-Alt-R); genespecific repair oligo $(4 \mu \mathrm{M})$; dpy-10 or unc-58 repair oligo $(1.34 \mu \mathrm{M})$; and Cas-9 protein $(24.5 \mu \mathrm{M})$. Wild-type germlines were injected to make puf-3(q966), puf-11 (q971), puf3(q1058), and puf-11(q1128). EG7866 [oxTi564 II; unc119(ed3) III] germlines were injected for dpy-10(q1074). $F_{1}$ progeny of injected hermaphrodites were screened for desired mutations by PCR and Sanger sequencing. Each allele was outcrossed against wild type at least twice before analysis.

\section{Isolation of puf-3 (q801)}

The puf-3(q801) 776 nucleotide deletion allele was isolated from a mutagenized library (gift of Maureen Barr), PCR screened to identify homozygotes, and outcrossed against wild type six times before analysis.

\section{RNA interference}

For RNA interference (RNAi), we identified clones targeting puf-3, puf-5, puf-6, puf-7, puf-8, and puf-9 from the Ahringer library (Fraser et al. 2000). The library does not include a clone targeting puf-11, so we generated one using the Gibson assembly method (Gibson 2009). Nucleotides $1-800$ of the puf-11 ORF were amplified from C. elegans complementary DNA and cloned into the plasmid L4440 at the NcoI site. For all RNAi experiments, the L4440 plasmid lacking a gene of interest ("empty" RNAi) served as a control.

To perform RNAi by feeding (Timmons and Fire 1998), E. coli HT115(DE3) were transformed with RNAi vectors and cultured at $37^{\circ}$ overnight in $2 \times$ YT media containing $25 \mu \mathrm{g} / \mu \mathrm{l}$ carbenicillin and $50 \mu \mathrm{g} / \mu \mathrm{l}$ tetracycline. Bacterial cultures were concentrated and seeded onto NGM plates containing $1 \mathrm{mM}$ IPTG, then induced overnight at room temperature. We then placed mid-L4 hermaphrodites on these plates. For experiments shown in Figure 3, D and E, we assayed treated animals $48 \mathrm{hr}$ after plating. For all other experiments, treated 
A Germline stem cell (GSC) regulatory pathway

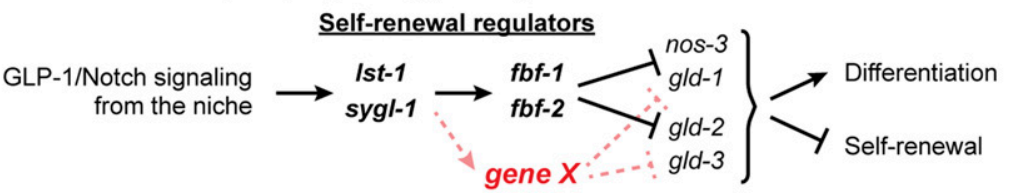

B

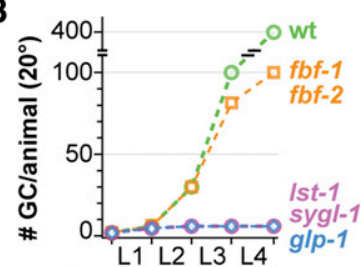

Developmental stage

C

\begin{tabular}{lcccccc} 
& \multicolumn{6}{c}{ \# GC/animal (mean $\pm \mathbf{s d}$ ) } \\
\cline { 2 - 7 } Genotype & $\mathbf{1 5 ^ { \circ }}$ & $\mathrm{n}$ & $\mathbf{2 0 ^ { \circ }}$ & $\mathrm{n}$ & $\mathbf{2 5 ^ { \circ }}$ & $\mathrm{n}$ \\
\hline glp-1 & $4 \pm 2$ & 20 & $3 \pm 2$ & 10 & $4 \pm 1$ & 16 \\
Ist-1 sygl-1 & $6 \pm 1$ & 10 & $5 \pm 1$ & 20 & $4 \pm 2$ & 10 \\
fbf-1 fbf-2 & $91 \pm 20$ & 16 & $128 \pm 30$ & 20 & $\begin{array}{c}\text { many \& } \\
\text { still dividing }\end{array}$ & 50 \\
\hline
\end{tabular}

D

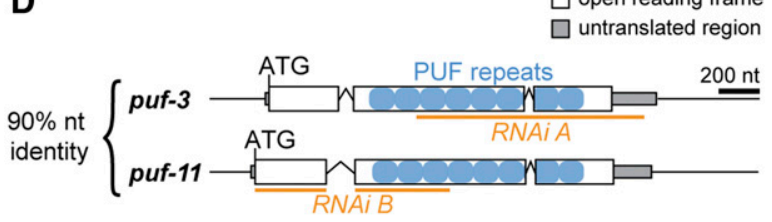

$\mathbf{E}$
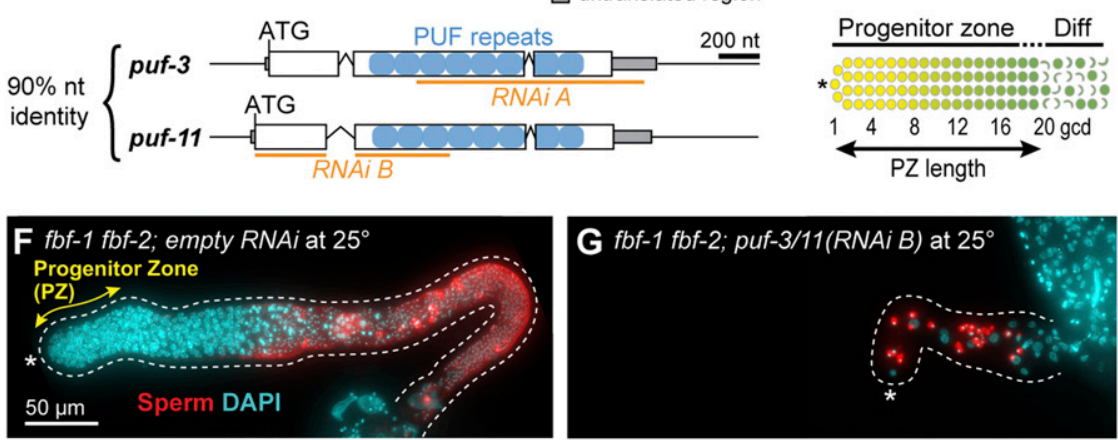

\begin{tabular}{|c|c|c|c|c|}
\hline H & & $\begin{array}{r}\text { Dist } \\
a\end{array}$ & $\begin{array}{l}\text { germ } \\
\text { Its at } 2\end{array}$ & \\
\hline Genotype \& Sex & RNAi & $\begin{array}{l}\% \\
\mathrm{PZ}\end{array}$ & $\begin{array}{c}\% \\
\text { sperm }\end{array}$ & $\mathrm{n}$ \\
\hline & empty & 100 & 0 & 21 \\
\hline$f b f-1$ fbf-2 이 $^{7}$ & puf-3/11 A & 13 & 87 & 15 \\
\hline & puf-3/11 B & 0 & 100 & 19 \\
\hline & empty & 97 & 3 & 30 \\
\hline$f b f-1$ fbf-2; him-5 $\sigma^{7}$ & puf-3/11 A & 0 & 100 & 8 \\
\hline & puf-3/11 B & 18 & 82 & 22 \\
\hline
\end{tabular}

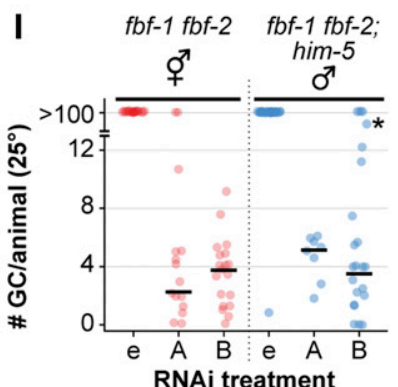

Figure 1 puf-3 and puf-11 are putative missing GSC regulators. (A) Diagram illustrating functional relationships between key genes that regulate stem cell self-renewal and differentiation in C. elegans germline stem cells (GSCs) (Kimble and Crittenden 2007; Kershner et al. 2014). Gene X (red) represents a missing factor that likely functions in parallel to $f b f-1$ and $f b f-2$. (B) Total number of germ cells (GCs) per animal at $20^{\circ}$ in wild type (wt) and strong lossof-function mutants of GSC regulators: $f b f-1 \mathrm{fbf}-2$, Ist-1 sygl-1, and g/p-1; data adapted from the literature (Austin and Kimble 1987; Crittenden et al. 2002, 2006; Kimble and Crittenden 2005; Kershner et al. 2014). x-axis: L1-L4, larval stages of development; ticks mark molts between stages. (C) Total GC number produced per animal; data generated in this work. Animals of each strain were staged to $36 \mathrm{hr}\left(15^{\circ}\right), 24 \mathrm{hr}\left(20^{\circ}\right)$, or $18 \mathrm{hr}\left(25^{\circ}\right)$ past mid-L4 for this experiment. In germlines where distal germ cells had differentiated to mature sperm, GC number was determined by counting sperm number and dividing by four. GC number were not counted in larger germlines that retained a PZ; these were scored as "many and still dividing." $n$ indicates the number animals scored. Alleles used here and throughout this work are strong loss of function and likely null: g/p-1(q46), Ist-1(q869), sygl-1(q828), fbf-1(ok91), and fbf-2(q704). (D) Schematic of puf-3 and puf-11 loci, which share 90\% nucleotide (nt) identity (Hubstenberger et al. 2012): exons (white boxes), introns (peaked lines), untranslated region (gray boxes), sequence encoding individual PUF repeats (blue circles), and sequence targeted by RNAi clone (orange line). (E) Diagram of distal gonad with the PZ and germ cells that have entered early meiotic prophase (Diff). Distal end (asterisk); GSCs (yellow); GSC daughters primed for differentiation and transitioning toward entry into meiotic prophase, including those in meiotic $S$ phase (graded yellow to green); and early meiotic prophase (green, crescent-shaped). ( $F$ and $\mathrm{G})$ Representative images of extruded gonads from $25^{\circ} \mathrm{fbf}-1 \mathrm{fbf}-2$ adult hermaphrodites staged to

$18 \mathrm{hr}$ after L4. RNAi treatment, either empty vector control (F) or puf-3/11 RNAi clone B (G), was initially delivered to these animal's mothers and then continued from hatching, through larval development and into adulthood. Gonads are immunostained with a sperm marker $\alpha$-SP56 (red) and DAPI (cyan). Images are Z-projections of several fluorescent images obtained on a compound microscope. A dotted line delineates the gonad boundary and an asterisk marks the distal end. Double-headed arrow (yellow) indicates the PZ. Scale bar in F applies to both images. (H) State of distal germ cells of $f b f-1$ $\mathrm{fbf}-2$ hermaphrodites and fbf-1 fbf-2; him-5 males, raised at $25^{\circ}$ on either empty or puf-3/11 RNAi, and staged to adulthood (18 hr after L4). Distal germ cell states were determined by DAPI-stained chromosomal morphology, scoring for a PZ or mature sperm in the distal germline. him-5(e1490) increased the frequency of male progeny (Hodgkin et al. 1979). (I) Quantitation of total GC per animal from experiment in $\mathrm{H}$ by counting sperm number and dividing by four. In germlines with a PZ, GC numbers were not counted but appeared comparable to previous reports of >100 (Merritt and Seydoux 2010; Shin et al. 2017). RNAi treatment on $x$-axis: empty vector (e), puf-3/11 RNAi clone A (indicated by A), or puf-3/11 RNAi clone B (indicated by B). Individual data points are plotted as circles; middle line is median value. * marks an animal with a total of $47 \mathrm{GCs}$.

animals were allowed to lay eggs and their $\mathrm{F}_{1}$ progeny were assayed for defects. All RNAi experiments were performed at least twice with controls and generated reproducible results; all images shown in the figures are representative.

\section{Analysis and quantification of germ cells in adults}

We first staged animals to roughly the same stage of adulthood. Specifically, we picked mid-L4s raised at 15,20 , or $25^{\circ}$ for at least one generation and grew them for an additional $18 \mathrm{hr}\left(25^{\circ}\right), 24 \mathrm{hr}\left(20^{\circ}\right)$, or $36 \mathrm{hr}\left(15^{\circ}\right)$ to synchronize adults for analysis. Whole worms were then stained with 4',6-diamidino-2-phenylindole (DAPI) and imaged by compound microscopy. The presence or absence of a progenitor zone (PZ) was assayed by nuclear morphology of germ cells at the distal end of the gonad: those lacking meiotic nuclear morphology and often possessing $\mathrm{M}$ phase nuclei were scored as PZ-positive (see also PZ analysis, below); those having a meiotic prophase nuclear morphology were scored as "meiotic" and PZ-negative; arms where all germ cells had differentiated as mature sperm were scored as "sperm" and 
A
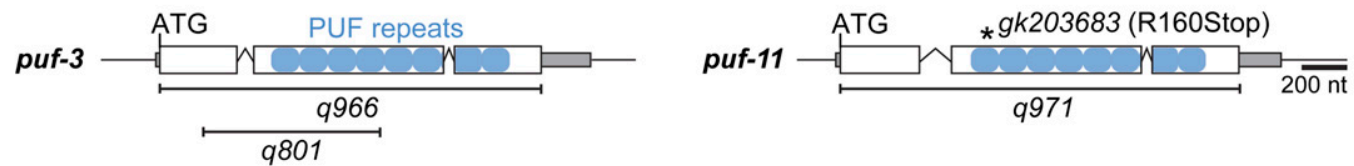

\begin{tabular}{|c|c|c|c|c|c|c|c|c|}
\hline $\begin{array}{l}\text { B } \\
\text { Genotype }\end{array}$ & $\%$ fertile & e $n$ & $\begin{array}{c}\text { \# embryos laid } \\
\text { mean } \pm \text { sd }\end{array}$ & $\mathrm{n}$ & $\begin{array}{c}\% \text { embro viability } \\
\text { mean (range) }\end{array}$ & $\mathrm{n}$ & $\begin{array}{c}\mathrm{PZ} \text { length (gcd) } \\
\text { mean } \pm \text { sd }\end{array}$ & $n$ \\
\hline wild-type & 99 & 196 & $264 \pm 72$ & 11 & $95(75-100)$ & 2901 & $19 \pm 2$ & 21 \\
\hline puf-3(q966) & 98 & 95 & $217 \pm 60$ & 10 & $95(84-100)$ & 2174 & $18 \pm 1$ & 10 \\
\hline puf-3(q801) & 100 & 86 & $215 \pm 40$ & 10 & $92(20-100)$ & 2153 & $20 \pm 2$ & 10 \\
\hline puf-11(q971) & 97 & 97 & $240 \pm 136$ & 10 & $43(0-59)$ & 2397 & $18 \pm 3$ & 10 \\
\hline puf-11(gk203683) & 100 & 102 & $294 \pm 40$ & 5 & $90(86-94)$ & 1472 & $19 \pm 2$ & 10 \\
\hline puf-3(q966) puf-11(q971) & 100 & 102 & $125 \pm 87$ & 10 & $0(0-1)$ & 1254 & $20 \pm 2$ & 19 \\
\hline puf-3(q801) puf-11(gk203683) & 100 & 101 & $102 \pm 40$ & 12 & $0(0-1)$ & 1218 & $20 \pm 3$ & 20 \\
\hline
\end{tabular}
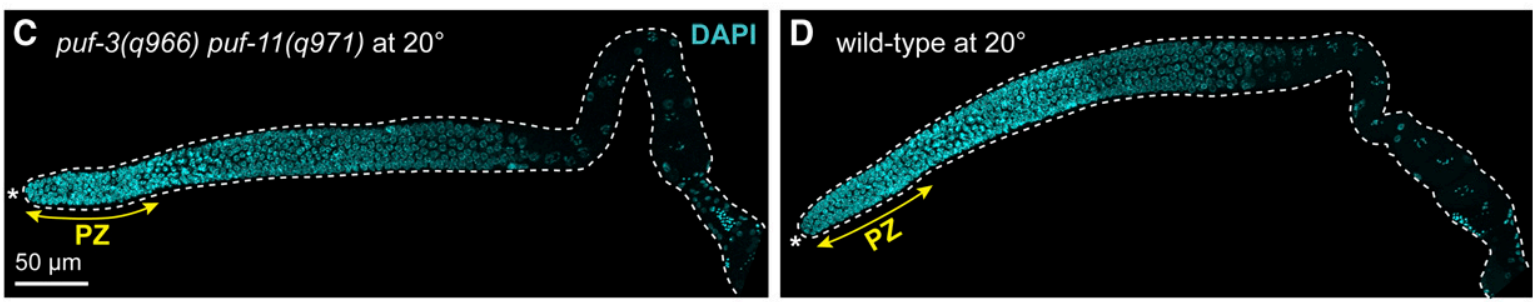

\begin{tabular}{|c|c|c|}
\hline \multirow{2}{*}{$\begin{array}{l}\text { E } \\
\text { Genotype }\end{array}$} & \multicolumn{2}{|c|}{$\begin{array}{c}\# \mathbf{G C} \text { in } \mathbf{P Z} \\
\text { mean } \pm \text { sd, } n=5\end{array}$} \\
\hline & $20^{\circ}$ & $25^{\circ}$ \\
\hline wild-type & $212 \pm 55$ & $191 \pm 20$ \\
\hline puf-3(q966) puf-11(q971) & $216 \pm 23$ & $176 \pm 24$ \\
\hline puf-3(q801) puf-11(gk203683) & $254 \pm 17$ & $171 \pm 50$ \\
\hline
\end{tabular}

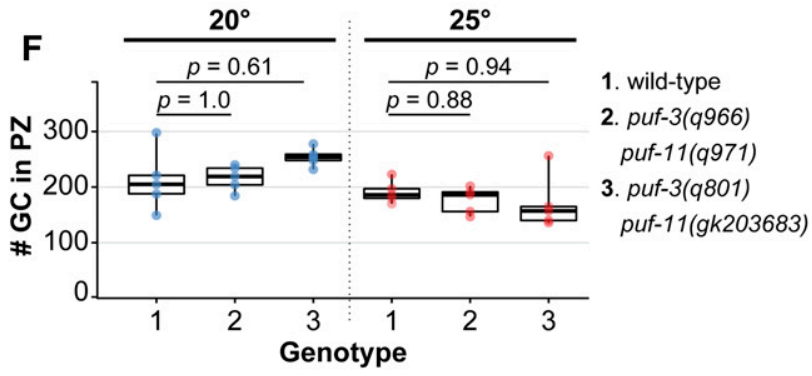

Figure 2 GSC maintenance defects are undetectable in single and double puf-3 and puf-11 mutants. (A) puf-3 and puf-11 loci, using conventions as in Figure 1D. Extents of deletion mutants are bracketed below loci and position of the point mutation is marked with an asterisk above. See Figure S2 for sequence details. (B) Germline-related characteristics of single and double puf-3 and puf-11 mutants at $20^{\circ}$. See Materials and Methods for details about assays and scoring. (C and D) Representative confocal Z-projections of DAPI-stained gonads extruded from animals staged to $24 \mathrm{hr}$ after L4 at $20^{\circ}$. Extent of PZ, double-headed yellow arrow. Annotation by convention in Figure 1, F and G and scale bar in C applies to D. (E and F) PZ sizes measured in number of germ cells (GCS). (F) PZ sizes showing individual data points as circles; middle line, median; boxes, 25-75\% quantile; whiskers, minimum and maximum values. $n=5$ gonad arms for each sample. $P$-values: mutants were compared to wild type using Welch's ANOVA and Games-Howell post hoc test.

PZ-negative. To estimate total germ cell number, we counted mature sperm (which have a distinctive, compact DAPI morphology) using the Multipoint Tool in Fiji/ImageJ (Schindelin et al. 2012) and then divided sperm number by four (each germ cell makes four sperm). In some cases, we counted sperm number in one gonadal arm and multiplied that number by two to estimate total sperm number. In cases where all germ cells in an animal had not yet differentiated to sperm, we did not count total germ cells.

\section{Phenotype analyses: fertility, brood size, and embryo viability}

L4 hermaphrodites were placed onto individual plates at $20^{\circ}$. At 6- to 24-hr intervals, each hermaphrodite was moved to a new plate and the embryos were counted to score for fertility and determine brood size. Several days later, hatched progeny on each plate were counted to determine embryo lethality.

\section{PZ analysis}

From roughly staged adults as described above, gonads were extruded, stained with DAPI, and imaged by compound or confocal microscopy. We examined morphology of germline nuclei to determine PZ size according to convention (Crittenden et al. 2006; Seidel and Kimble 2015) (see also Figure 1E). Briefly, DAPI staining of nuclei in early meiotic prophase adopts a crescent shape. To count number of germ cells in a PZ, we counted the total number of cells in the distal 
A Triple mutants

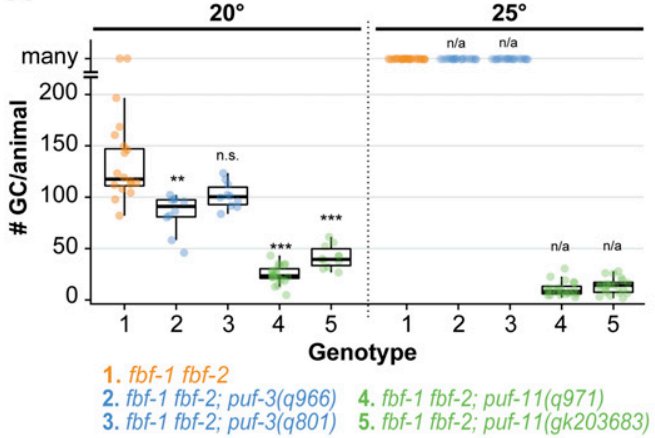

B

\begin{tabular}{lcccr} 
& \multicolumn{4}{c}{ Distal germ cells, } \\
\cline { 2 - 6 } adults at 25 \\
\cline { 2 - 6 } Genotype & $\begin{array}{c}\% \\
\%\end{array}$ & $\begin{array}{c}\% \\
\text { meiotic }\end{array}$ & sperm & $\mathrm{n}$ \\
\hline$f b f-1$ fbf-2 & 97 & 3 & 0 & 100 \\
\hline$f b f-1$ fbf-2; puf-3(q966) & 34 & $\mathbf{6 4}$ & 2 & 85 \\
$f b f-1$ fbf-2; puf-3(q801) & 23 & $\mathbf{7 6}$ & 1 & 100 \\
\hline$f b f-1$ fbf-2; puf-11(q971) & 0 & 0 & $\mathbf{1 0 0}$ & 100 \\
$f b f-1 ~ f b f-2 ;$ puf-11(gk203683) & 0 & 0 & $\mathbf{1 0 0}$ & 100 \\
\hline
\end{tabular}

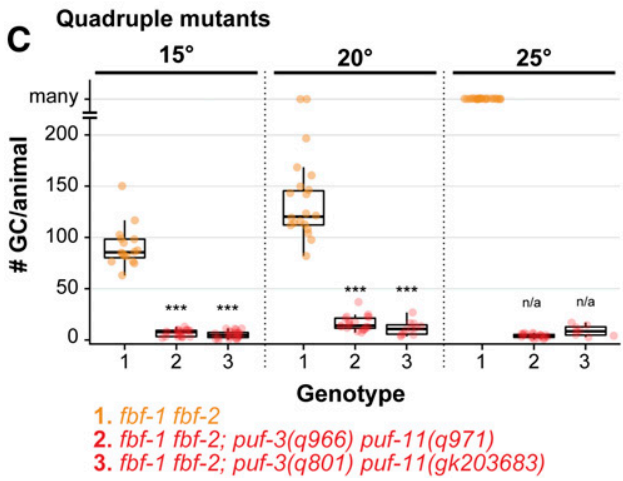

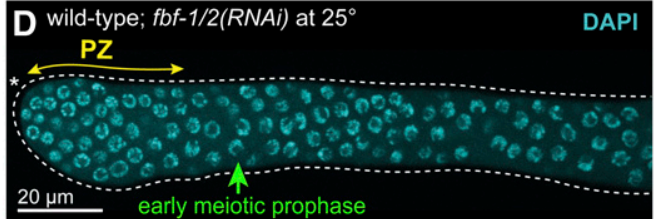

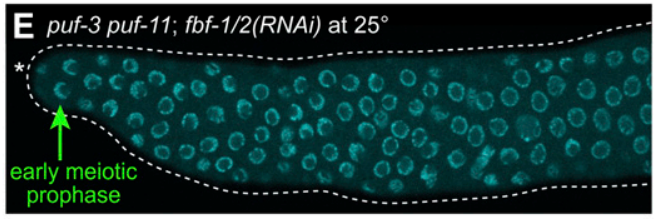

Figure 3 Triple and quadruple mutants reveal puf-3 and puf-11 role in GSC maintenance. (A) Total germ cell (GC) number per animal in fbf-1 fbf-2; puf triple mutant adults at $20^{\circ}$ (left) and $25^{\circ}$ (right). GC number was counted only in germlines differentiated to the distal end; germlines retaining a PZ or having meiotic prophase nuclear morphology were scored as "many." Where distal germ cells had differentiated to mature sperm, GC number was determined by counting sperm and then dividing by four. Individual data points are plotted as circles; middle line, median; boxes, $25-75 \%$ quantile; whiskers, minimum and maximum values falling outside the box but within 1.5 times the interquartile range. To determine $P$-values, triple mutants were compared to $f b f-1$ fbf-2 control using Welch's ANOVA and Games-Howell post-hoc test; n.s., not significant; ** $P<0.01$, $* * * P<0.001 ; \mathrm{n} / \mathrm{a}$, not applica-

ble. For additional information including mean GC/animal, see Figure S3. (B) State of distal germ cells in $f b f-1 \mathrm{fbf}-2 ;$ puf triple mutants at $25^{\circ}$, assayed in adults at $18 \mathrm{hr}$ past L4. State was scored as described in Figure $1 \mathrm{H}$, with the additional classification of "meiotic" for cells with meiotic prophase chromosomal morphology, but not yet differentiated as sperm. (C) Graph of germ cell number per animal in fbf-1 fbf-2; puf-3 puf-11 adults at 15 , 20 and $25^{\circ}$. Germline scoring and graph conventions as in A. P-value compared to respective $f b f-1 \mathrm{fbf}-2$ control was determined using Welch's ANOVA and Games-Howell post-hoc test; ***P<0.001; n/a, not applicable. For additional information including mean GC per animal, see Figure S3. (D and E) Representative images of DAPI-stained gonads extruded from wild type (D) or puf-3(q966) puf-11(q971) (E) adult hermaphrodites treated with fbf-1/2 RNAi at $25^{\circ}$. Animals were plated to RNAi as mid-L4s and analyzed for changes in nuclear morphology $48 \mathrm{hr}$ later, when the RNAi treatment had had time to work effectively and animals had reached adulthood. The extent of the PZ (double-headed arrow, yellow) and "crescent-shaped nuclei" typical of early meiotic prophase (green) are annotated. Other annotation by convention in Figure 1, F and G. Images show single confocal Z-sections and scale bar in D applies to both images.

germline that had not entered early meiotic prophase, using Fiji/Image J Cell Counter plugin (Schindelin et al. 2012). We also measured the distance to the end of the PZ in germ cell diameters from the distal end. To do this, we selected a middle focal plane and counted the number of germline nuclei along each edge of the gonad until the first one with crescent morphology. We averaged the two values from each edge to determine PZ size.

\section{Quantification of germ cells in larvae}

To generate roughly synchronous embryos, we allowed gravid hermaphrodites to lay eggs for $2 \mathrm{hr}$ at $20^{\circ}$. At subsequent timepoints corresponding to early L1, early L2, late L2, and early L4, larvae were harvested and germ cell number was quantitated. For early L1s, we scored germ cell number in live animals by DIC microscopy. For all remaining samples, we used whole-mount staining with the reduction/oxidation method (Finney and Ruvkun 1990; Miller and Shakes 1995). Briefly, samples were fixed in Ruvkun fixation buffer with $1 \%(\mathrm{w} / \mathrm{v})$ paraformaldehyde for $30 \mathrm{~min}$. Disulfide linkage reduction was performed, then samples were incubated in blocking solution [PBS with $1 \%(\mathrm{w} / \mathrm{v})$ bovine serum albumin, 0.5\% (v/v) Triton X-100, $1 \mathrm{mM}$ EDTA] for $40 \mathrm{~min}$ at room temperature, followed by overnight incubation at $4^{\circ}$ with rabbit $\alpha$-PGL-1 (1:100, gift from Susan Strome, University of California, Santa Cruz, CA) diluted in blocking solution. Secondary Alexa Fluor 555 donkey $\alpha$-rabbit (1:1000, \#A31570; Thermo Fisher Scientific) antibody was diluted in blocking solution and incubated with samples for at least $1 \mathrm{hr}$ along with $1 \mathrm{ng} / \mu 1$ DAPI for DNA visualization. Samples were mounted in Vectashield (\#H1000; Vector Laboratories, Burlingame, CA) on $2 \%$ agarose pads, then assayed by fluorescence compound microscopy. The number of germ cells in L2s was determined by counting the total number of PGL-1positive cells. For early L4s, we counted the number of PGL-1positive cells in one gonadal arm then multiplied by two to estimate the total number of germ cells per animal.

\section{Immunostaining and DAPI staining}

We immunostained gonads as described (Crittenden et al. 2017 ) with minor modifications. To extrude gonads, we dissected animals in PBS buffer with $0.1 \%(\mathrm{v} / \mathrm{v})$ Tween-20 (PBStw) and $0.25 \mathrm{mM}$ levamisole. Gonads were fixed in $3 \%(\mathrm{w} / \mathrm{v})$ paraformaldehyde diluted in PBStw for $10 \mathrm{~min}$, 


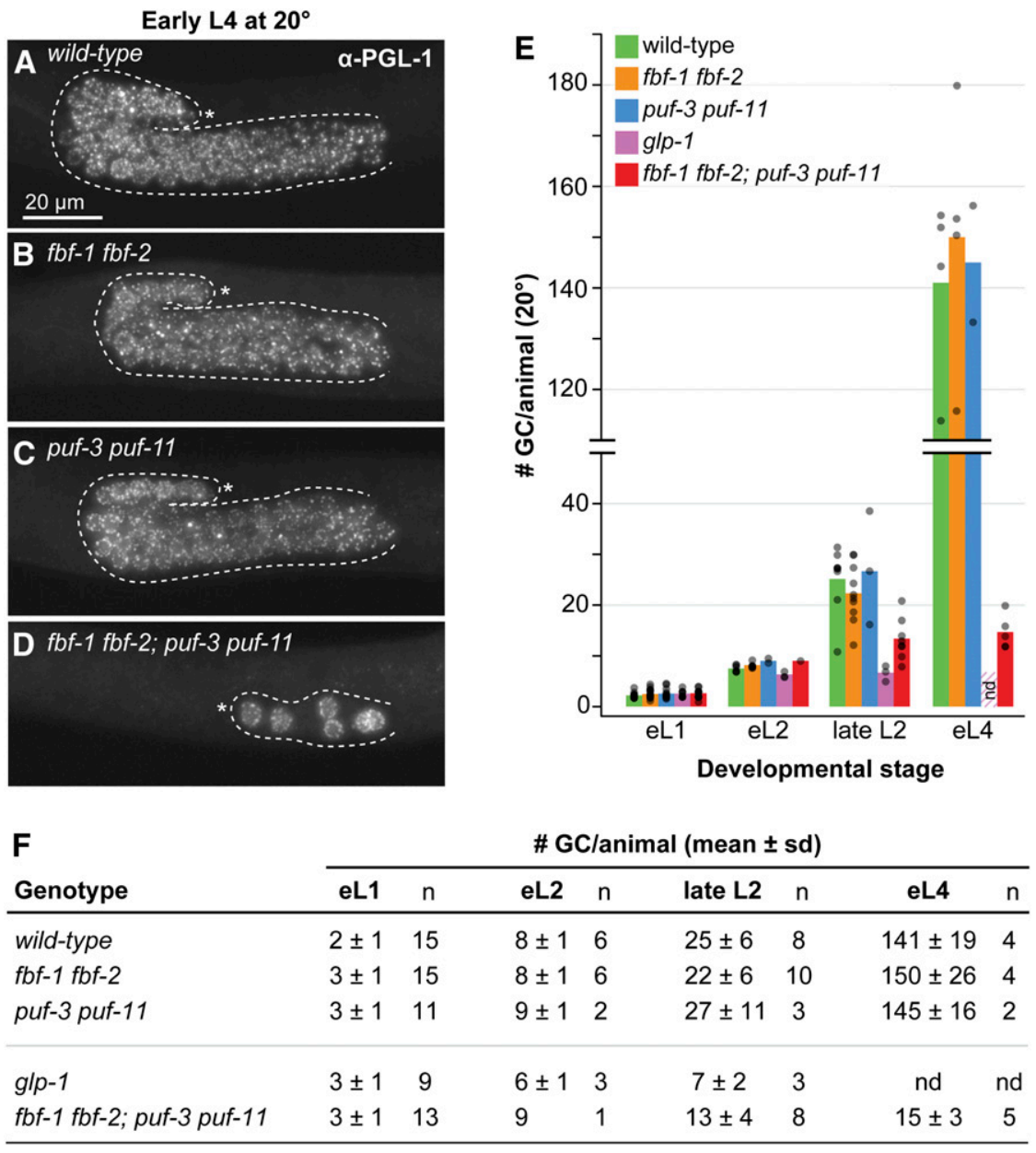

Figure 4 GSC self-renewal fails during larval development in $\mathrm{fbf-1} f \mathrm{fb}-2$; puf-3 puf-11 quadruple mutants at $20^{\circ}$. (A-D) Representative images of early L4 germlines stained with $\alpha$-PGL-1 (white), a germ cell marker. Images were obtained by fluorescence compound microscopy. The scale bar in A applies to all images. All other annotation by convention in Figure 1, F and $G$. ( $E$ and $F$ ) Quantitation of the number of germ cells (GCS) per animal across larval stages. Larvae were immunostained (as in A-D) at defined developmental timepoints: early L1 (eL1), early L2 (eL2), late L2, and early L4 (eL4) stages. GC number was counted as number of PGL-1-positive cells for all timepoints, except early L1, which was scored live by DIC microscopy. (E) Bars show the mean number of germ cells per sample with genotypes color coded as shown. Each individual data point is plotted as a gray circle. not done (nd): germ cells in g/p-1 animals at early L4 stage had already differentiated to sperm and were thus not scored; the striped bar in early L4 is replicated from the late L2 mean value for $g / p-1$. (F) Table with mean number of GCs per animal. (A-F) Alleles are as follows: fbf-1(ok91), fbf-2(q704), puf-3(q966), puf-11(q971), g/p-1(q46). then permeabilized in either $0.2 \%(\mathrm{v} / \mathrm{v})$ Triton-X diluted in PBStw or ice-cold methanol for 10-15 min. Next, gonads were incubated for at least $1 \mathrm{hr}$ in blocking solution $[0.5 \%$ $(\mathrm{w} / \mathrm{v})$ bovine serum albumin diluted in PBStw] and incubated overnight at $4^{\circ}$ with the following primary antibodies diluted in blocking solution: mouse $\alpha$-V5 (1:1000, SV5-Pk1, \#MCA1360; Bio-Rad, Hercules, CA) and mouse $\alpha$-SP56 (1:200, gift from Susan Strome, University of California, Santa Cruz, CA). Secondary antibody Alexa Fluor 488 donkey $\alpha$-mouse (1:1000, \#A21202; Thermo Fisher Scientific) was diluted in blocking solution and incubated with samples for at least $1 \mathrm{hr}$. To visualize DNA, DAPI was included with the secondary antibody at a final concentration of $1 \mathrm{ng} / \mu \mathrm{l}$. Samples were mounted in Vectashield (\#H1000; Vector Laboratories) or ProLong Gold (\#P36930; Thermo Fisher Scientific) before imaging. All steps were performed at room temperature unless otherwise indicated. Where only DNA visualization was required, we skipped all blocking solution steps and instead simply incubated samples in PBStw with $1 \mathrm{ng} / \mu \mathrm{l}$ DAPI for 15 min before mounting.

\section{Microscopy}

Images in Figure 2, C and D, Figure 3, D and E, Figure 5, B-G, Figure S1, A and B, and Figure S4, B-J were taken using a laser scanning Leica TCS SP8 confocal microscope fitted with both photomultiplier and hybrid detectors, and run using LAS software version X. All images were taken with a $63 \times$ / 1.40 NA CS2 HC Plan Apochromat oil immersion objective at $400 \mathrm{~Hz}$ scanning speed and $125-200 \%$ zoom. To prepare images for figures, Adobe Photoshop was used to equivalently and linearly adjust intensity among images to be compared.

Images in Figure 1, F and G, Figure 4, A-D, and Figure S5 were captured using a Hamamatsu ORCA-Flash4.0 cMOS camera on a Zeiss Axioskop compound microscope equipped with a 63/1.4 NA Plan Apochromat oil immersion objective. The fluorescent light source was a Lumencore SOLA Light Engine, and Carl Zeiss filter sets 49 and 38 were used for DAPI and Alexa 488 visualization. The acquisition software was Micromanager (Edelstein et al. 2010, 2014). When required, images were combined using the Pairwise Stitching function in Fiji/ImageJ (Preibisch et al. 2009). To prepare images for figures, Adobe Photoshop was used to adjust intensity equivalently and linearly among images to be compared.

\section{Fluorescence quantitation}

Quantitation of fluorescence in Figure 5, H and I was performed with Fiji/ImageJ (Schindelin et al. 2012). In Figure 

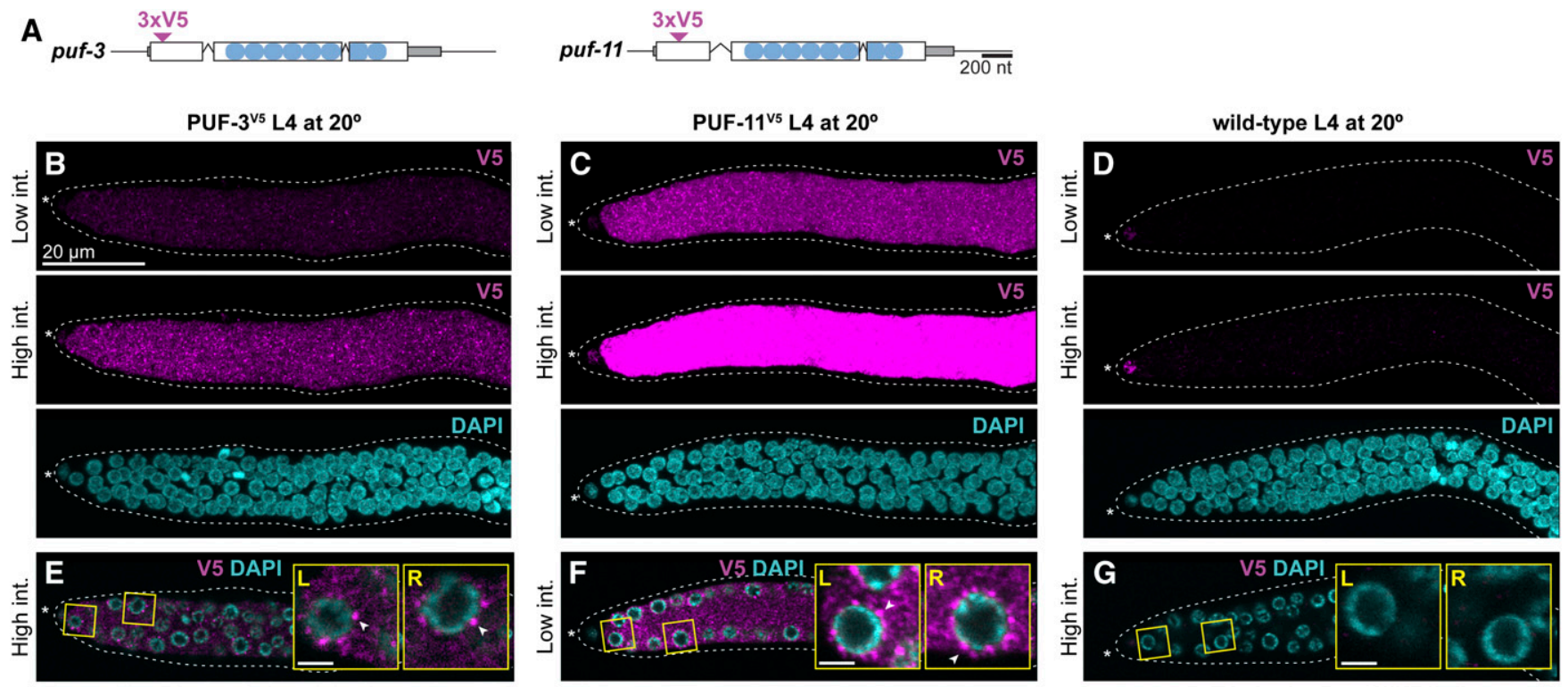

\section{H Distal expression in L4s}

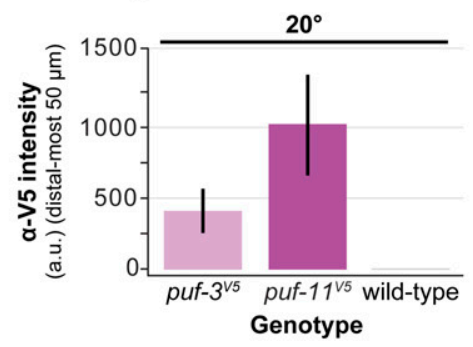

\section{Distal expression in adults}

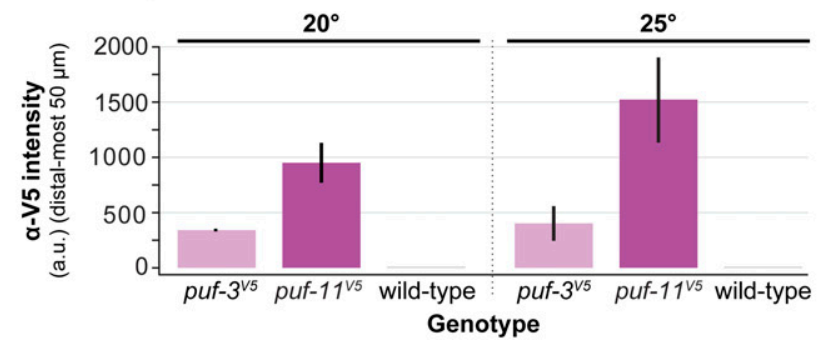

Figure 5 PUF-3 and PUF-11 are expressed in distal germline, including GSCs. (A) Schematic of puf-3 and puf-11 loci with epitope tags annotated by convention as in Figure 1D. Inverted triangles denote insertion sites of 3XV5 epitope tags, which are flanked both up- and downstream by a GS linker. See Figure S2 for a more detailed sequence annotation. (B-G) Representative images of PUF- $3^{\vee 5}$ and PUF-1 $11^{\vee 5}$ expression in gonads extruded from L4 hermaphrodites raised at $20^{\circ}$. Gonads from puf-3V5 [puf-3(q1058)] (B and E), puf-11 V5 [puf-11(q1128)] (C and F), and wild-type control (D and G) were stained with $\alpha$-V5 (magenta) and DAPI (cyan) and then imaged by confocal microscopy. (B-D) are Z-projections. V5 signal intensity (int.) was adjusted uniformly in Adobe Photoshop across images, and high or low intensities are indicated on the left. In all genotypes, including the wild-type control lacking a V5 epitope, nonspecific staining was detected in the somatic distal tip cell with the $\alpha-V 5$ antibody. In E-G, a middle Z-slice from the respective gonad in B-D is shown. Magnified insets are outlined in yellow and labeled left $(L)$ or right $(R)$ corresponding to their position in the image; white arrowheads mark representative cytoplasmic granules. Scale bar in B applies to all images except the insets, where Bar, $2 \mu \mathrm{m}$. Other annotation conventions as in Figure 1, F and G. (H and I) Quantitation of V5 intensity in the distal region of PUF-3V5 (light pink), PUF-11 155 (dark pink), and wild-type (gray) extruded gonads, determined using Fiji/lmageJ (see Materials and Methods for details). Each bar represents the mean $\alpha$-V5 immunostaining intensity in arbitrary units (a.u.) in the distal-most $50 \mu \mathrm{m}$ of the gonad [ 11 germ cell diameters (gcd) using the conversion $4.4 \mu \mathrm{m} / \mathrm{gcd}$ (Lee et al. 2016)], with nonspecific staining background from the wild-type control subtracted. Error bars represent SE. (H) Analysis of mid-L4 staged extruded germlines at $20^{\circ}$. Four independent experiments were performed for a total of at least 27 gonads per genotype. (I) Analysis of germlines extruded from staged adults at $20^{\circ}\left(24 \mathrm{hr}\right.$ past mid-L4) and $25^{\circ}(18 \mathrm{hr}$ past mid-L4). Two independent experiments were performed for a total of 24 gonads per experimental condition. For representative images at $20^{\circ}$, see Figure S4, E-G.

$5 \mathrm{H}$, we performed four independent immunostaining experiments and quantitated a total of at least 27 gonads per genotype. In Figure 5I, we performed two independent experiments and quantitated at least 24 gonads per genotype. From confocal image stacks, we collected raw pixel intensity data from each gonad image by projecting the sum of all $Z$-slices onto a single plane. A freehand line, 50 pixels wide and $50 \mu \mathrm{m}$ long, bisecting bisected the gonad, was drawn manually using the Plot Profile tool starting at the distal tip of the tissue. We found the mean intensity value of the plot profile of each gonad to get a single value reflecting the amount of protein present. Next, we subtracted any signal representing nonspecific antibody binding for each independent experiment: we calculated the mean intensity value in the respective wild-type control gonads, then subtracted it from each experimental sample. In Figure 5, $\mathrm{H}$ and I, we report the background subtracted mean and SE.

\section{Genetic epistasis experiments}

To test the relationship between $l s t-1$ sygl- 1 and $f b f-1 f b f-2$; puf-3 puf-11, we used transgenes that ubiquitously express LST- 1 and SYGL-1 protein. Because these lst- 1 (gf) and sygl1 (gf) transgenes cause germline tumors and are sterile, they were maintained on $l s t-1$ or sygl-1 RNAi, respectively, before 


\begin{tabular}{|c|c|c|c|c|c|c|}
\hline \multirow{2}{*}{ A } & \multirow[b]{2}{*}{ RNAi } & \multicolumn{3}{|c|}{ Germline phenotype } & \multicolumn{2}{|c|}{ \# GC/animal } \\
\hline & & $\%$ pGIp 1 & $\% G \mid p^{2}$ & $\mathrm{n}$ & mean $\pm \mathrm{sd}$ & $n$ \\
\hline \multirow{2}{*}{ Ist-1(gf); fbf-1 fbf-2 } & empty & 100 & 0 & 106 & $\mathrm{n} / \mathrm{a}$ & $\mathrm{n} / \mathrm{a}$ \\
\hline & puf-3/11 & 24 & 76 & 29 & $5 \pm 3$ & 22 \\
\hline \multirow{2}{*}{$s y g l-1(g f) ; f b f-1$ fbf-2 } & empty & 100 & 0 & 87 & $\mathrm{n} / \mathrm{a}$ & $\mathrm{n} / \mathrm{a}$ \\
\hline & puf-3/11 & 17 & 83 & 24 & $3 \pm 2$ & 20 \\
\hline
\end{tabular}

1. pGlp, partial Glp phenotype; germ cell number similar to $f b f-1 \mathrm{fbf}-2$ double mutant.

2. Glp, tiny germlines with only a few sperm; germ cell number similar to g/p-1 null.

\begin{tabular}{llccc} 
& & \multicolumn{3}{c}{ Germline phenotype } \\
\cline { 3 - 5 } Genotype & RNAi & $\%$ tumor $^{1}$ & $\%$ Glp $^{2}$ & $\mathrm{n}$ \\
\hline \multirow{2}{*}{ gld-1 gld-2; fbf-1 fbf-2 } & empty & $\mathbf{1 0 0}$ & 0 & 25 \\
& puf-3/11 & $\mathbf{1 0 0}$ & 0 & 28 \\
\hline
\end{tabular}

1. Tumorous germlines are vastly overproliferating.

2. Glp, tiny germlines with only a few sperm; germ cell number similar to g/p-1 null.

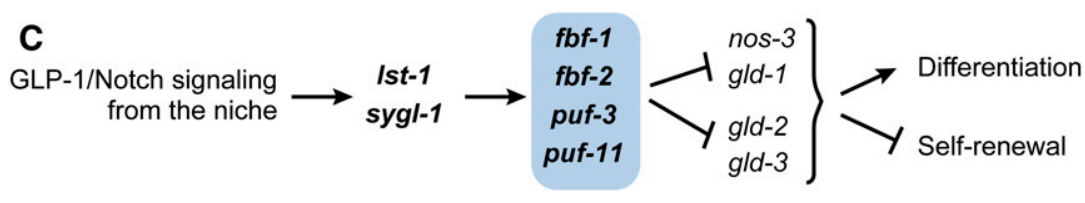

Figure 6 puf-3 puf-11 lie parallel to $f b f-1 f b f-2$ in the GSC regulatory pathway. (A) Results of epistasis tests conducted with 1 st-1 (gf) and sygl-1(gf) alleles at $25^{\circ}$. Number of germ cells (GCs) in Glp animals was determined by counting sperm in adults and dividing by four. Genotype for Ist-1(gf) strain is Ist-1(ok814); qSi267 [Pmex-5::LST-1::3xFLAG::tbb-2 3' end] fbf-1 (ok91) fbf2(q704) and sygl-1 (gf) is sygl-1(tm5040); qSi235 [Pmex5::SYGL-1::3xFLAG::tbb-2 3' end] fbf-1(ok91) fbf2(q704). We used the puf-3/11 RNAi clone $B$ in these experiments. Images of representative germlines are available in Figure S5, A-D. (B) Results of epistasis tests conducted with $\mathrm{g} / \mathrm{d}-1 \mathrm{~g} / \mathrm{d}-2$ at $20^{\circ}$. Genotype is g/d2(q497) gld-1 (q361); fbf-1 (ok91) fbf-2(q704). We used the puf-3/11 RNAi clone B in these experiments. Images of representative germlines are available in Figure S5, E and F. (C) Revised pathway for GSC regulation that includes puf-3 and puf-11 at the same position in the pathway as $f b f-1$ and $f b f-2$ (blue shading). the experiment. Expression of LST-1 or SYGL-1 was then induced by transferring L4s to OP50-seeded NGM plates and passaging for several generations (Shin et al. 2017). The assay in Figure 6A and Figure S5, A-D was performed at $25^{\circ}$. From populations grown for nine generations on OP50, we plated L4s onto puf-3/11 RNAi. Next, progeny of puf-3/11 RNAi-treated animals were staged to $24 \mathrm{hr}$ past L4 and stained with DAPI to assay effects on germline development. Because $l s t-1$ and sygl-1 require $f b f-1$ fbf- 2 for function, neither $l s t-1$ ( $g f$ ) nor sygl-1 (gf) formed a germline tumor in these experiments.

To test the relationship between $g l d-2$ gld -1 and $f b f-1 f b f-2$; puf-3 puf-11 for Figure 6B and Figure S5, E-G, we plated mid-L4 staged JK5778 [gld-2(q497) gld-1(q361)/ccIs4251 unc-15(e73) I; fbf-1(ok91) fbf-2(q704)/mIn1[mIs14 dpy$10(e 128)] \mathrm{II}]$ to $\mathrm{puf}-3 / 11 \mathrm{RNAi}$ plates at $20^{\circ} . \mathrm{F}_{1}$ progeny were staged to 24 hr past L4, then stained with DAPI and imaged using compound microscopy to assay germline phenotype.

\section{Yeast two-hybrid}

Modified yeast two-hybrid assays were performed as described (Bartel and Fields 1997). PUF proteins were fused to the LexA DNA binding domain as follows: complementary DNA sequences encoding PUF repeats of FBF-1 (aa 121614), FBF-2 (aa 121-632), PUF-3 (aa 88-502), PUF-9 (aа 162-703), and PUF-11 (aa 91-505) were each cloned into the NdeI site of pBTM116 using the Gibson assembly method (Gibson et al. 2009). We also used full-length LST-1 (aa 1328) and SYGL-1 (aa 1-206) fused to Gal4 activation domain in the pACT2 vector (Shin et al. 2017). More details about plasmids are available in Table S3. To test for protein-protein interactions between PUFs and LST-1/SYGL-1, activation and binding domain pairs were cotransformed into a L40-ura3 strain [MATa, ura3-52, leu2-3,112, his3 200 , trp1 1 1, ade2, LYS2::(LexA-op) 4 -HIS3, ura3::(LexA-op) 8 -LacZ] using the lithium acetate method (Gietz and Schiestl 2007). LacZ reporter activity was measured using the Beta-Glo Assay system (\#E4720; Promega, Madison, WI), following commercial protocols and yeast-specific methods (Hook et al. 2005). Luminescence was quantitated using a Biotek Synergy H4 Hybrid plate reader with Gen5 software.

\section{Western blots}

For the Western blots in Figure 7C, we grew yeast transformants in -Leu -Trp liquid media and prepared samples by boiling 0.5 OD of yeast in sample buffer (60 mM Tris, $\mathrm{pH} 6.8$, $25 \%$ glycerol, $2 \%$ SDS, $0.1 \%$ bromophenol blue with $14 \mathrm{mM}$ beta-mercaptoethanol or $100 \mathrm{mM}$ DTT). Analysis was conducted on a 4-15\% SDS-PAGE gradient gel (\#456-1083; Bio-Rad). We probed with primary antibodies overnight at $4^{\circ}$ as follows: mouse anti-HA (1:50,000, HA.11, \#MMS101R; Covance), mouse anti-V5 (1:1000, SV5-Pk1, \#MCA1360; Bio-Rad ), or mouse anti-actin (1:10,000, C4, \#MAB1501; Millipore, Bedford, MA). For secondary antibodies, blots were incubated for $1-2 \mathrm{hr}$ at room temperature with 1:20,000 donkey anti-mouse horseradish peroxidase (\#715035-150; Jackson ImmunoResearch). Immunoblots were developed using SuperSignal West Pico/Femto Sensitivity substrate (\#34080, \#34095; Thermo Scientific) and a Konica Minolta SRX-101A medical film processor. For final figure preparations, intensity of the blot was linearly adjusted in Adobe Photoshop.

\section{Statistical analysis}

Welch's ANOVA and Games-Howell post hoc tests were performed to calculate statistical significance for multiple 
A

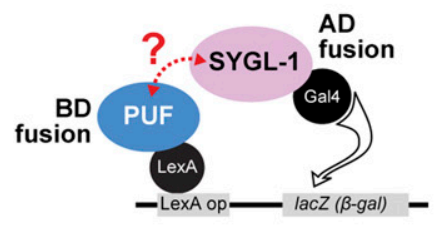

B

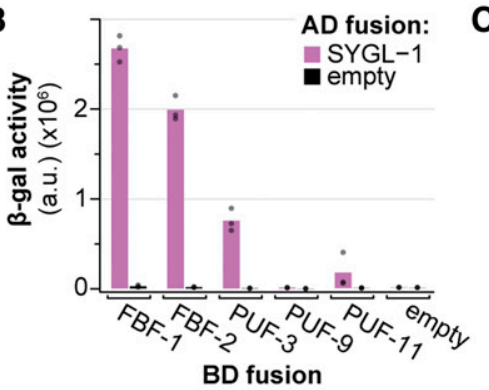

C AD fusion: SYGL-1
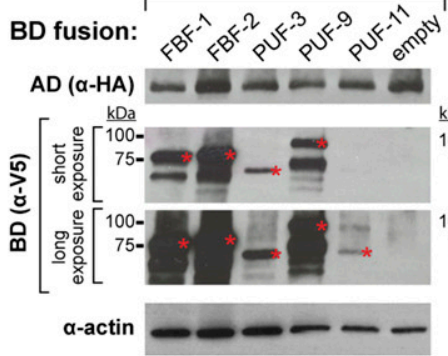

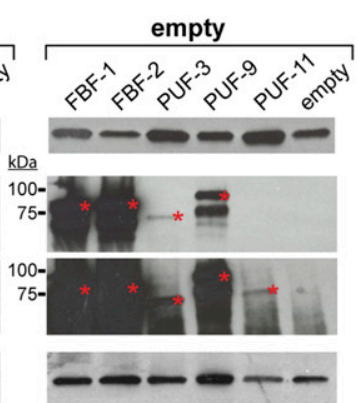

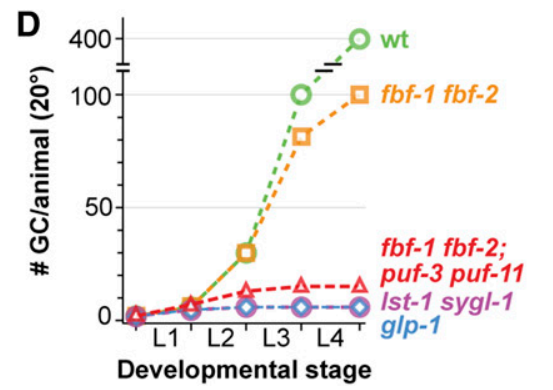

E

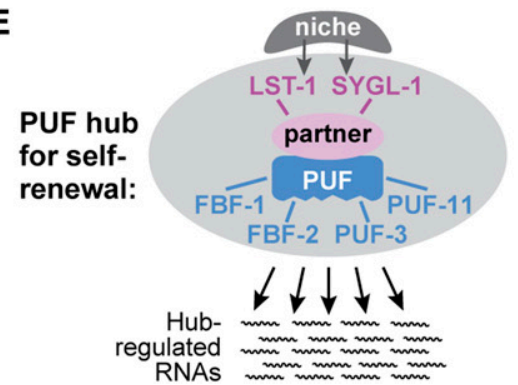

F Molecular model for GSC self-renewal:

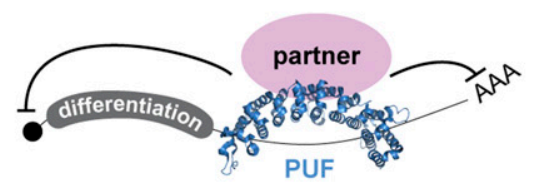

Figure 7 PUFs and FBF comprise a PUF hub that accounts for GSC self-renewal. (A) Yeast two-hybrid schematic. SYGL-1 was fused to the Gal4 activation domain (AD), which was HA tagged. PUF protein variants were fused to the LexA binding domain (BD), which was V5 tagged. PUF constructs included the PUF repeats and some flanking amino acids; for amino acid boundaries, see Materials and Methods. Interaction between SYGL-1 and PUF drives transcription of a lacZ ( $\beta$-gal) reporter. (B) SYGL-1/PUF interaction was measured using $\beta$-galactosidase ( $\beta$-gal) activity. Each bar is the mean of at least three independent experiments. Individual data points are plotted as gray circles. (C) Western blot from yeast lysate probed with $\alpha$-HA to detect AD fusion proteins and $\alpha-V 5$ to detect BD fusion proteins. For the blots detecting BD::V5::PUF fusions, the molecular weight ladder is annotated at left and a red asterisk marks the relevant full-length protein in each lane. Expected sizes are as follows: FBF-1, 80.9 kDa; FBF-2, 82.4 kDa; PUF-3, 71.9 kDa; PUF-9, $85.9 \mathrm{kDa}$; and PUF-11, $71.7 \mathrm{kDa}$. Actin was the loading control. (D) Total number of germ cells (GCs) per animal at $20^{\circ}$ in mutants of key GSC regulators, modified from Figure $1 \mathrm{~B}$ to include $\mathrm{fbf-1} f b f-2 ;$ puf-3 puf-11 (red triangles) (this work). (E) The PUF hub for GSC self-renewal consists of two PUF partners that are direct targets of niche signaling and four PUF RNA-binding proteins that collectively regulate a battery of messenger RNAs. ( $F$ ) Molecular model for GSC self-renewal: PUF RNA-binding protein binds to the 3' UTR of target messenger RNAs. A PUF partner, LST-1 or SYGL-1, ensures RNA repression by an unknown mechanism.

samples. All statistical tests were performed in $\mathrm{R}$ and the $P$-value cut off was 0.05 .

\section{Data and reagent availability}

The authors affirm that all data necessary for confirming the conclusions of this article are present within the article, tables, figures, and supplemental material. All protocols are available upon request. All strains and plasmids are available upon request or via the Caenorhabditis Genetic Center, supported by the National Institutes of Health Office of Research Infrastructure Programs (P40 OD010440). Supplemental material available at figshare: https://doi.org/10.25386/ genetics.10250801.

\section{Results}

\section{Solidifying evidence for the existence of gene $X$}

The existence of a missing GSC self-renewal regulator, gene X, was proposed because of striking differences in GSC defects upon removal of either the GLP-1/Notch receptor or its target genes (lst-1 and sygl-1) on the one hand and removal of FBF-1 and FBF-2 on the other (Figure 1B) (Austin and Kimble 1987; Crittenden et al. 2002; Kershner et al. 2014). To provide comprehensive data as a critical baseline for this study, we scored GSC defects in key mutants at 15, 20, and $25^{\circ}$ (Figure 1C). As expected, mutants lacking GLP-1/Notch or both its target genes, lst-1 sygl-1, generated only $\sim 4$ germ cells at each temperature, but $f b f-1 f b f-2$ double mutants made many more (Figure 1C). In addition, $f b f-1 f b f-2$ mutants raised at $25^{\circ}$ possessed distal germ cells in mitotic metaphase or anaphase, consistent with active divisions (Figure S1, A and B). A previous study showed that these distal cells express a mitotic marker, nuclear REC-8 [see Shin et al. (2017), Figure S5G]. Thus, loss of FBF-1 and FBF-2 has a much less severe and later effect on GSC maintenance than loss of niche signaling or loss of the niche targets, confirming the notion of some missing self-renewal regulator (gene X, Figure 1A).

\section{PUF-3 and PUF-11 proteins are likely the missing self-renewal regulators}

To begin our search for the missing GSC regulators, we considered other PUF proteins as logical candidates. Among 10 C. elegans PUF-encoding genes (Liu et al. 2012), PUF-11 piqued our interest because of two similarities with FBF: the PUF-11 protein interacts with LST-1 in a genome-wide yeast two-hybrid screen (Boxem et al. 2008), and its RNA-binding specificity is similar to that of FBF (Bernstein et al. 2005; Koh et al. 2009). Because the C. elegans genome encodes a PUF-11 
paralog with nearly identical sequence, called PUF-3 (Figure 1D and Figure S2), we tested both for a role in GSC maintenance. To this end, we used two feeding RNAi clones, one targeting puf-3 (RNAi A) and the other targeting a distinct region of puf-11 (RNAi B) (Figure 1D). Although these RNAi clones target different gene regions, each was expected to deplete both puf-3 and puf-11 because of their 90\% nucleotide identity (Hubstenberger et al. 2012).

A previous puf-3/11 RNAi study performed in wild-type animals identified a role for PUF-3 and PUF-11 in oogenesis, but not GSC maintenance (Hubstenberger et al. 2012). We therefore sought a more GSC-specific assay and turned to enhancement of the $f b f-1 f b f-2$ pGlp phenotype at $25^{\circ}$. For this analysis, we scored the presence or absence of a PZ, the distal region where germ cells have not yet entered into the meiotic cell cycle and continue mitotic divisions (Figure 1E). Whereas all $f b f-1 f b f-2$ adults on empty vector RNAi possessed a PZ at $25^{\circ}$ (Figure $1, \mathrm{~F}$ and $\mathrm{H}$ ), most treated with puf-3/11 RNAi lost their PZ to differentiation (Figure 1, G and $\mathrm{H}$ ). A comparable effect was seen in both sexes (Figure $1 \mathrm{H})$. Strikingly, GSC divisions generated only $\sim 4$ germ cells per gonad in each sex (Figure 1I), as determined by counting the number of mature sperm in adults and dividing by four. RNAi directed against other puf loci (e.g., puf-8) did not enhance the pGlp $f b f-1 f b f-2$ phenotype (Figure S1C). Therefore, $p u f-3 / 11$ RNAi enhances the $f b f-1 f b f-2$ germline phenotype from its partial pGlp to the full Glp typical of GLP-1/Notch mutants. This enhancement indicates that PUF-3 and PUF-11 are likely the missing self-renewal regulators.

\section{puf-3 and puf-11 mutants have no GSC proliferation defects on their own}

The puf-3/11 RNAi experiments could not distinguish between the puf-3 and puf-11 genes for effects on GSC maintenance. To test their individual roles, we analyzed puf-3 and puf-11 single mutants: three deletions, puf-3(q801), puf3(q966), and puf-11(q971), and one nonsense allele, puf11 ( $g k 203683$ ) (Figure 2A and Figure S2). As a measure of general germline function, we scored fertility, number of embryos laid, and embryo viability. The single mutants were fertile with brood sizes comparable to wild type, and their embryos hatched into young larvae, except for puf-11(q971), which had a partially penetrant embryonic lethality (Figure 2B). In addition, we scored PZ lengths as a proxy for effects on GSCs. The PZ lengths, measured with the conventional metric of germ cell diameters from the distal end, were roughly the same as wild type in all puf-3 and puf-11 single mutants (Figure 2B). Therefore, puf-3 and puf-11 single mutants have no major GSC proliferation defects.

We next assessed puf-3 puf-11 double mutants (Figure 2, B-F). Germlines had an organization and size comparable to wild type (Figure 2, C and D), but they made no viable embryos (Figure 2B). Male puf-3 puf-11 crossed to feminized fog-1 hermaphrodites yielded ample cross progeny, so puf-3 puf-11 sperm are functional. By contrast, wild-type males crossed to puf-3 puf-11 hermaphrodites failed to make viable progeny, suggesting that puf-3 puf-11 oocytes are defective, consistent with prior studies (Hubstenberger et al. 2012, 2013). PZ lengths were comparable to wild type, scored at $20^{\circ}$ (Figure 2B), and number of germ cells therein were comparable to wild type at both 20 and $25^{\circ}$ (Figure 2, E and F). Therefore, puf-3 puf-11 double mutants have an oogenesis defect, as previously recognized, but no obvious GSC defects.

\section{puf-3 and puf-11 enhancement of fbf-1 fbf-2 partial Glp phenotype}

To further explore puf-3 and puf-11 roles in GSC maintenance, we tested for enhancement of the $f b f-1 \quad f b f-2$ pGlp phenotype using $f b f-1 f b f-2$; puf triple mutants as well as $f b f-1$ fbf-2; puf-3 puf-11 quadruple mutants. To score enhancement, we determined total germ cells made in each strain by counting mature sperm number in adults and dividing by four; at $25^{\circ}$, we scored for the persistence of a $\mathrm{PZ}$ in adults.

We first assayed triple mutants raised at $20^{\circ}$ (Figure 3A, left, and Figure S3). The control fbf-1 fbf-2 double mutants made roughly 100 germ cells before GSCs were lost to spermatogenesis, as previously described (Crittenden et al. 2002; Lamont et al. 2004; this work). That number decreased in both triple mutants, but the extent of pGlp enhancement differed for puf-3 and puf-11 (Figure 3A, left, and Figure S3). The decrease in germ cell number was small in $f b f-1$ $f b f-2$; puf-3 triple mutants and statistically significant for only one allele; the decrease was larger in $f b f-1$ fbf-2; puf-11 mutants and statistically significant for both alleles (Figure 3A, left, and Figure S3). Nonetheless, each puf gene contributed to larval GSC proliferation at $20^{\circ}$.

We also assayed triple mutants raised at $25^{\circ}$. The control $f b f-1$ fbf- 2 double mutants made $>100$ germ cells and maintained a PZ, as described previously (Merritt et al. 2008; Shin et al. 2017; this work). In $f b f-1 f b f-2$; puf-3 triple mutants, germline size was comparable to $f b f-1 \mathrm{fbf}-2$ double mutants (Figure 3A, right, and Figure S3), but many PZs were lost to meiotic entry (Figure 3B). By contrast, $f b f-1 f b f-2$; puf-11 triple mutants at $25^{\circ}$ made far fewer germ cells overall than $f b f-1 f b f-2$ (Figure 3A, right, and Figure S3), and had a fully penetrant PZ loss with all germ cells differentiating as sperm (Figure 3B). Thus, each puf gene enhanced the $f b f-1 f b f-2$ pGlp defect at $25^{\circ}$, with puf-11 again having a more severe effect than puf-3.

We next assayed $f b f-1$ fbf-2; puf-3 puf-11 quadruple mutants, this time raised at 15,20 , or $25^{\circ}$. The two distinct quadruple mutants, fbf-1 fbf-2; puf-3(q966) puf-11(q971) and $f b f-1 f b f-2$; puf-3 (q801) puf-11 (gk203683), were remarkably similar at all three temperatures. Adults had tiny germlines composed entirely of mature sperm. Upon quantitation, quadruples made a total of four to nine germ cells on average at 15 and $25^{\circ}$, and $11-16$ at $20^{\circ}$ (Figure $3 \mathrm{C}$ and Figure S3). The quadruple Glp phenotype is comparable to $g l p-1$ and $l s t-1$ sygl-1 null mutants and is thus indicative of GSC failure to self-renew at an early larval stage (compare Figure S3 to 
Figure 1C). Thus, PUF-3 and PUF-11 function during larval development to maintain GSC divisions.

Finally, we sought to test the importance of PUF-3 and PUF-11 specifically in adult GSCs. The Glp phenotype in quadruple mutants (see above) demonstrated that PUF-3 and PUF-11 function in larval GSCs but did not address the possibility of a later role. We therefore asked if PUF-3 and PUF-11 play a role in maintaining the PZ in $f b f-1 f b f-2$ double mutant adults at $25^{\circ}$. We treated mid-L4 puf-3(q966) puf-11 (q971) double mutants with $f b f-1 / 2$ RNAi and assayed PZ presence or absence in adults (48 hr later). Most wild-type animals treated with $f b f-1 / 2$ RNAi retained a PZ $(92 \%, n=12)$ (Figure 3D). However, few puf-3 puf-11 double mutants treated with $f b f-1 /-2$ RNAi retained a PZ (7\%, $n=30$ ) (Figure 3E); instead, distalmost germ cells entered early meiotic prophase, visualized by a nuclear "crescent" morphology. Thus, PUF-3 and PUF-11 function in $25^{\circ} \mathrm{fbf}-1 \mathrm{fbf}-2$ adults to maintain a PZ.

\section{GSC maintenance fails during early larval development in quadruple mutants}

Up to this point, germ cell counts were performed in adults by counting sperm. This approach quantitates the number of cells generated and differentiated, but cannot detect cells that die without differentiation into gametes. Although germ cell death was not seen in $g l p-1$ or $l s t-1$ sygl-1 mutants (Austin and Kimble 1987; Kershner et al. 2014), the fbf-1 fbf-2; puf-3 puf-11 quadruple mutant may be different.

To begin, we assessed overall germline sizes at the L4 stage, which were comparable in wild type, $f b f-1 f b f-2$, and puf-3 puf-11, but much smaller in quadruple mutants (Figure 4, A-D). Next, we counted total germ cell number at specific intervals during larval development. For this experiment, we scored $f b f-1$ fbf-2; puf-3 puf-11 quadruple mutants and several controls (wild type, fbf-1 fbf-2, puf-3 puf-11, and $g(p-1)$, all maintained at $20^{\circ}$. PGL-1 staining was used to identify germ cells for counting (except for early L1, which was scored by DIC microscopy in live animals). We found that germ cell numbers increased similarly during larval development in wild type, $f b f-1$ fbf-2, and puf-3 puf-11 animals, but they did not increase appreciably in $g l p-1$ or the $f b f-1 f b f-2$; puf-3 puf-11 quadruple mutant (Figure 4, E and F). By L4, the quadruple mutants had made a total of 15 germ cells on average (range $=12-20, n=5$ ) (Figure 4, $\mathrm{E}$ and $\mathrm{F}$ ), similar to the number of germ cells estimated from adult sperm number at the same temperature (Figure 3C and Figure S3) and consistent with cell death having little effect on germ cell number. In parallel, we visualized meiotic entry with DAPI staining. By late L2, germ cells in quadruple mutants had entered meiotic prophase, whereas wild type, $f b f-1 f b f-2$, and puf-3 puf-11 had not. No morphological sign of germ cell death was seen over the course of these experiments. Together, these findings allay the concern that puf-3 puf-11 mutants might reduce germ cell number by promoting cell death and thus support the conclusion that puf-3 and puf- 11 normally promote self-renewal during larval development.

\section{PUF-3 and PUF-11 expression in GSCS}

To test whether PUF-3 and PUF-11 proteins are expressed in GSCs, we generated V5 epitope-tagged alleles (Figure 5A and Figure S2). Both PUF- $3^{\mathrm{V} 5}$ and PUF- $11^{\mathrm{V} 5}$ are functional, as assayed by their lack of $f b f$ enhancement (Figure S4A). Upon immunostaining and imaging, PUF- $3^{\mathrm{V} 5}$ and PUF- $11^{\mathrm{V} 5}$ proteins were observed in the distal germlines of mid-L4 hermaphrodites raised at $20^{\circ}$ (Figure 5, B-D and Figure S4, B-D for full gonad), of adult hermaphrodites raised at $20^{\circ}$ (Figure S4, E-G) and of mid-L4 males raised at $20^{\circ}$ (Figure S4, H-J). In addition, both proteins were present more proximally in developing oocytes (Figure S4, E-G). As expected for PUF proteins, both PUF- $3^{\mathrm{V} 5}$ and PUF- $11^{\mathrm{V} 5}$ were cytoplasmic (Figure 5, E-G) and localized to perinuclear granules (Figure 5, E and F, insets), consistent with an RNA regulatory role. $\alpha$-V5 staining was absent in wild-type germlines, as expected because they lacked the epitope tag. We quantified the PUF- $3^{\mathrm{V} 5}$ and PUF- $11^{\mathrm{V} 5}$ signal in the distal gonads of L4s and adults at $20^{\circ}$ (Figure 5, $\mathrm{H}$ and I), subtracting the very low background in wild type for each. PUF-11 was more abundant than PUF-3 at both stages. Finally, we confirmed expression of both proteins in adult distal germlines at $25^{\circ}$ and again quantitated the signal (Figure 5I). We conclude that PUF-3 and PUF-11 are expressed in GSCs.

\section{puf-3 puf-11 placement in GSC regulatory pathway}

The notion that puf-3 and puf-11 represent the missing selfrenewal regulators, dubbed gene $\mathrm{X}$, predicts their placement in the GSC regulatory pathway (Figure 1A). Their $f b f$ enhancement, reported above, is consistent with puf-3 puf-11 functioning in parallel to $f b f-1 f b f-2$, but we conducted two additional epistasis experiments to solidify that pathway position. For these experiments, we used RNAi to deplete puf-3 and puf-11, both for ease of genetic manipulation and because GSC defects were comparable after RNAi and in quadruple mutants.

We first investigated the relationship between puf-3 puf-11 and $l s t-1$ sygl- 1 . Previous studies showed that $f b f-1 f b f-2$ functions either downstream or in parallel to lst-1 sygl-1. This pathway placement was deduced using gain-of-function (gf) mutants of $l s t-1$ and sygl-1. Both $l s t-1$ (gf) and sygl-1 (gf) make massive germline tumors when $f b f-1$ and $f b f-2$ are wild-type, but acquire a pGlp phenotype when $f b f-1$ and $f b f-2$ are removed in $l s t-1(g f) ; f b f-1 f b f-2$ and sygl-1 (gf); fbf-1 fbf-2 triple mutants (Shin et al. 2017). To ask if puf-3 and puf-11 have the same genetic relationship to lst-1 and sygl-1, we treated lst-1 (gf); fbf-1 fbf-2 and sygl-1 (gf); fbf-1 fbf-2 triple mutants with either empty vector RNAi as a control or puf-3/11 RNAi. The control germlines had a pGlp phenotype (Figure 6A and Figure S5, A and C), as shown previously (Shin et al. 2017). However, with puf-3/11 RNAi, most germlines had a fully Glp phenotype, typical of $f b f-1 f b f-2$; puf-3 puf-11 quadruple mutants (Figure 6A). Their germlines were tiny with only a few sperm (Figure S5, B and D), and upon quantitation, only four to eight germ cells were made on average per animal (Figure 6A). Therefore, puf-3 and puf-11 likely function downstream or in parallel to lst-1 and sygl-1 (Figure 6C). 
We next investigated the relationship between puf-3 puf-11 and gld-1 gld-2. The gld-1 and gld-2 genes promote meiotic entry and in their absence, the germline becomes tumorous (Kadyk and Kimble 1998). Previous studies showed that $f b f-1$ fbf-2 functions upstream of gld-1 gld-2. Thus, gld-1 gld-2 tumors are epistatic to $f b f-1 f b f-2$ pGlp (Eckmann et al. 2004). To ask if puf-3 and puf-11 are similarly upstream of gld-1 gld-2, we treated gld-1 gld-2; fbf-1 fbf-2 quadruple mutants with puf-3/11 RNAi. Indeed, gld-1 gld-2 tumors were still found, demonstrating gld-1 gld-2 epistasis over fbf-1 fbf-2; puf-3/11 RNAi (Figure 6B and Figure S5, E and F). We confirmed that puf-3,11 RNAi knockdown was successful by comparison with embryos of wild-type siblings (Figure S5G). Therefore, puf-3 and puf-11 likely function upstream of gld-1 gld-2 (Figure 6C). Together, these experiments place puf-3 and puf-11 into the GSC regulatory pathway in a position consistent with their proposed identity as gene $X$.

\section{PUF-3 interacts with SYGL-1 in yeast}

Placement of puf-3 puf-11 alongside $f b f-1$ fbf-2 in the genetic pathway (above), together with their molecular identity as PUF RNA-binding proteins, suggests that PUF-3 and PUF-11 may have molecular activities in GSCs similar to FBF. Previous studies showed that FBF-1 and FBF-2 physically interact with Notch targets LST-1 and SYGL-1 (Shin et al. 2017; Qiu et al. 2019). Moreover, the LST-1 and SYGL-1 partnerships with FBF are essential in vivo for GSC self-renewal (Haupt et al. 2019; C. R. Kanzler and H. J. Shin, unpublished data). We therefore considered the possibility that PUF-3 and PUF-11 also form partnerships with LST-1 and SYGL-1. Indeed, a genome-wide interaction screen had already shown that an interaction between PUF-11 and LST-1 in yeast (Boxem et al. 2008). We therefore focused our yeast two-hybrid assays (Figure 7A) on interactions between PUF-3/PUF-11 and SYGL-1. FBF-1 and FBF-2 were tested as positive controls, and PUF-9 as a likely negative control. PUF-3 interacted robustly with SYGL-1, but PUF-9 and PUF-11 did not (Figure 7B). The discrepancy between PUF-3 and PUF-11 was initially confounding given the similarity of the two proteins. However, a Western blot revealed that PUF-11 was poorly expressed in yeast (Figure 7C). We conclude that PUF-3 interacts with SYGL-1 and suggest that PUF-11 likely does as well due to the near identity between them. These data plus those of the genome-wide screen (Boxem et al. 2008) indicate that PUF-3 and PUF-11 likely interact physically with LST-1 and SYGL-1.

\section{Discussion}

\section{Missing self-renewal regulators are PUF-3 and PUF-11}

Despite over three decades of research defining the regulatory network that maintains C. elegans GSCs, key self-renewal regulators were clearly missing (see Introduction; Figure 1, A and B). The argument was simple: animals lacking GLP-1/ Notch signaling from the niche or lacking the two key GLP-1/ Notch targets, lst-1 and sygl-1, have a much earlier GSC defect than animals lacking the only other known self-renewal regulators, FBF-1 and FBF-2. Because LST-1 and SYGL-1 proteins interact physically with FBF-1 and FBF-2 (Shin et al. 2017; Haupt et al. 2019; Qiu et al. 2019) and that interaction is essential for GSC self-renewal (Haupt et al. 2019; C. R. Kanzler and H. J. Shin, unpublished data), we predicted that the missing regulators might be additional PUF RNA-binding proteins. Indeed, we report here that animals lacking four PUF proteins, PUF-3 and PUF-11 in addition to FBF-1 and FBF-2, exhibit the same early GSC defect as glp-1 null mutants (Figure 7D). The fbf-1 fbf-2; puf-3 puf-11 germ cell number is equivalent to that of $g l p-1$ null at 15 and $25^{\circ}$. At $20^{\circ}$, the quadruple mutant undergoes one additional GSC division, which is a slight difference (one temperature, one cell division) but may reveal the existence of additional minor regulators. Nonetheless, PUF-3 and PUF-11 are the major missing self-renewal regulators.

In the process of characterizing puf- 3 and puf-11 as selfrenewal regulators, we confirmed their primary role in oogenesis. Previous studies using RNAi directed against puf-3/11 had identified their function in oogenesis: namely, to produce viable embryos (Hubstenberger et al. 2012; Tsukamoto et al. 2017). Our work extends these previous studies in three ways. Using deletion mutants of each gene, we find that PUF-3 and PUF-11 act redundantly during oogenesis; using tagged versions of each protein, we show that both are expressed in oocytes; and using genetics, we find that PUF-3 and PUF-11 are not required for spermatogenesis. Importantly, while their oogenesis function is exclusive to hermaphrodites, their role in GSC self-renewal is critical in both hermaphrodites and males and thus is gender-independent. Their molecular mechanism of action in both GSCs and oocytes likely revolves around RNA regulation, a common theme among PUF proteins. Regardless, we emphasize that PUF-3 and PUF-11 are the long-sought missing self-renewal regulators.

\section{$A$ "PUF hub" is responsible for GSC self-renewal}

Regulatory networks are central to cell fates and tissue patterning across animal phylogeny. For germline fates and patterning, regulatory networks that rely on post-transcriptional regulation have emerged as particularly prominent (e.g., Kimble and Crittenden 2007; Slaidina and Lehmann 2014; Yamaji et al. 2017), with key RNA-binding proteins regulating hundreds of RNAs and directing cell fate programs (Kershner and Kimble 2010; Aoki et al. 2018; Porter et al. 2019; this work). This work reveals that PUF RNA-binding proteins are principal intrinsic regulators in the stem cell network. Four PUFs collectively drive GSC self-renewal in hermaphrodites and males, in larvae and adults and at all laboratory growth temperatures $\left(15,20\right.$, and $\left.25^{\circ}\right)$. The centrality of PUF proteins to the GSC regulatory network is underscored by the fact that GSC defects of $f b f-1$ fbf-2; puf-3 puf-11 quadruple null mutants are virtually identical to those of $g l p-1 /$ Notch null and $l s t-1$ sygl-1 null mutants (Figure 7D).

The remarkable phenotypic congruence of mutants in niche signaling, its targets LST-1 and SYGL-1, and four PUF 
proteins leads us to propose the concept of a "PUF self-renewal hub" in the stem cell regulatory network (Figure 7E). This hub consists of four PUF RNA-binding proteins and two PUF partner proteins, LST-1 and SYGL-1. LST-1 and SYGL-1 were first identified as partners of FBF (Shin et al. 2017; Haupt et al. 2019; Qiu et al. 2019), but PUF-3 and PUF-11 also likely partner with LST-1 and SYGL-1 (Boxem et al. 2008; this work). Moreover, these PUF partnerships are essential for GSC self-renewal (Haupt et al. 2019; C. R. Kanzler and H. J. Shin, unpublished data). The PUF hub therefore serves as the principal node for GSC self-renewal in the stem cell regulatory network.

The PUF hub seems remarkably simple and is strongly supported by genetic and molecular analyses. However, puzzles remain. For example, mutants in the fog-1 gene, which encodes a CPEB-related RNA-binding protein, also enhance the GSC defect of $f b f-1 f b f-2$ double mutants, but that enhancement is coupled to a reversal in germline sex and the mechanism remains a mystery (Thompson et al. 2005). In contrast, as emphasized here, the "PUF hub" GSC phenotype is not coupled to any effect on germline sex determination, but instead is equivalent to removal of niche signaling (Kershner et al. 2014; this work). Most other intrinsic stem cell regulators do not meet this high bar of equivalence to the niche-defective phenotype. Thus, the PUF hub promises to provide a paradigm for understanding self-renewal hubs more broadly.

\section{Redundancy and buffering within the PUF hub}

The PUF hub relies on a striking nexus of functional redundancies. PUF-3 and PUF-11 are redundant with FBF during larval development and in adults at $25^{\circ}$ (this work); and FBF-1 and FBF-2 are redundant with each other in late larval development at 15 and $20^{\circ}$ (Crittenden et al. 2002). Moreover, the two PUF partners, LST-1 and SYGL-1, are functionally redundant (Kershner et al. 2014; Shin et al. 2017). These layers of redundancy, together with our molecular understanding of individual hub proteins, suggests a simple molecular model (Figure 7F). In this model, each PUF protein binds to target RNAs via 3'UTR regulatory elements and also binds to either LST-1 or SYGL-1 to elicit RNA repression. Evidence for this model is particularly compelling for FBF, whose mode of action has been analyzed most intensively (Bernstein et al. 2005; Wang et al. 2009; Shin et al. 2017; Haupt et al. 2019). Data are also strongly suggestive for the nearly identical PUF-3 and PUF-11 proteins: PUF-11 binds to RNA with a sequence specificity similar to that of FBF (Koh et al. 2009); PUF-3/-11 repress expression of reporter RNAs in oocytes (Hubstenberger et al. 2012); PUF-11 interacts with LST-1 in yeast (Boxem et al. 2008); and PUF-3 interacts with SYGL-1 in yeast (this work). Although a rigorous characterization of the PUF/partner/RNA complex remains a critical direction for future studies, the architectural outlines and key molecular features of the hub are clear.

The extensive redundancy among hub components suggests that components are, to a first approximation, molecularly interchangeable. That interchangeability likely renders the hub robust, namely capable of maintaining stem cells under many conditions (e.g., developmental stage, growth temperature, sex). Although not yet analyzed, the interchangeability may also help stem cells withstand the barrage of environmental inputs and stresses experienced outside the laboratory. A similar phenomenon of functional redundancy of key regulators has been found in other developmental regulatory networks (e.g., Hox genes in animal development, MAD box genes in plant development) and likely lies at the heart of network evolution more broadly (Félix and Wagner 2008).

In addition to functional redundancy, single hub components likely have specialized individual roles. Intensive studies of FBF-1 and FBF-2 reveal numerous individual features (Lamont et al. 2004; Voronina et al. 2012; Voronina 2013; Brenner and Schedl 2016; Prasad et al. 2016; Wang et al. 2016; Porter et al. 2019). FBF-1 and FBF-2 have distinct low penetrance sex determination defects, genetic interactions, expression, subcellular localization, target RNAs, and FBF-specific molecular effects on targets. PUF-3 and PUF-11 will also likely possess differences, between each other and also with FBF. Understanding the common and unique roles among the members of the hub will be crucial to understanding how the hub is buffered to maintain stem cells under a variety of physiological and environmental conditions.

\section{Acknowledgments}

We thank Peggy Kroll-Conner for help generating strains central to this work as well as isolation of the puf-3(q801) deletion, Maureen Barr for sharing her mutagenesis library, and the Million Mutations Project for generating puf11 ( $g$ k203683). We thank Susan Strome (University of California, Santa Cruz, CA) for $\alpha$-PGL- 1 and $\alpha$-SP56 antibodies. We are also grateful to Sarah Crittenden and Brian Carrick for comments on the manuscript, Laura Vanderploeg for help with figures, and Carol Pfeffer for help with manuscript preparation. Some strains used in the study were provided by the Caenorhabditis Genetics Center, supported by the National Institutes of Health Office of Research Infrastructure Programs (P40 OD010440). This work was supported by the National Institutes of Health (GM050942 to M.W. and GM134119 to J.K.); J.K. was an investigator with the Howard Hughes Medical Institute. The authors declare no competing interests.

\section{Literature Cited}

Angelo, G., and M. R. Van Gilst, 2009 Starvation protects germline stem cells and extends reproductive longevity in C. elegans. Science 326: 954-958. https://doi.org/10.1126/ science. 1178343

Aoki, S. T., D. F. Porter, A. Prasad, M. Wickens, C. A. Bingman et al., 2018 An RNA-binding multimer specifies nematode sperm fate. Cell Rep. 23: 3769-3775. https://doi.org/10.1016/j.celrep.2018.05.095

Arribere, J. A., R. T. Bell, B. X. Fu, K. L. Artiles, P. S. Hartman et al., 2014 Efficient marker-free recovery of custom genetic modifications with CRISPR/Cas9 in Caenorhabditis elegans. Genetics 198: 837-846. https://doi.org/10.1534/genetics.114.169730 
Austin, J., and J. Kimble, $1987 g l p-1$ is required in the germ line for regulation of the decision between mitosis and meiosis in C. elegans. Cell 51: 589-599. https://doi.org/10.1016/00928674(87)90128-0

Bartel, P. L., and S. Fields (Editors), 1997 The Yeast Two-Hybrid System. Oxford University Press, New York.

Bernstein, D., B. Hook, A. Hajarnavis, L. Opperman, and M. Wickens, 2005 Binding specificity and mRNA targets of a C. elegans PUF protein, FBF-1. RNA 11: 447-458. https://doi.org/ 10.1261/rna.7255805

Boxem, M., Z. Maliga, N. Klitgord, N. Li, I. Lemmens et al., 2008 A protein domain-based interactome network for C. elegans early embryogenesis. Cell 134: 534-545. https://doi.org/10.1016/ j.cell.2008.07.009

Boyer, L. A., T. I. Lee, M. F. Cole, S. E. Johnstone, S. S. Levine et al., 2005 Core transcriptional regulatory circuitry in human embryonic stem cells. Cell 122: 947-956. https://doi.org/ 10.1016/j.cell.2005.08.020

Brenner, J. L., and T. Schedl, 2016 Germline stem cell differentiation entails regional control of cell fate regulator GLD-1 in Caenorhabditis elegans. Genetics 202: 1085-1103. https:// doi.org/10.1534/genetics.115.185678

Brenner, S., 1974 The genetics of Caenorhabditis elegans. Genetics 77: 71-94.

Crittenden, S. L., D. S. Bernstein, J. L. Bachorik, B. E. Thompson, M. Gallegos et al., 2002 A conserved RNA-binding protein controls germline stem cells in Caenorhabditis elegans. Nature 417: 660-663. https://doi.org/10.1038/nature754

Crittenden, S. L., K. A. Leonhard, D. T. Byrd, and J. Kimble, 2006 Cellular analyses of the mitotic region in the Caenorhabditis elegans adult germ line. Mol. Biol. Cell 17: 3051-3061. https://doi.org/10.1091/mbc.e06-03-0170

Crittenden, S. L., H. S. Seidel, and J. Kimble, 2017 Analysis of the C. elegans germline stem cell pool. Methods Mol. Biol. 1463: 133. https://doi.org/10.1007/978-1-4939-4017-2_1

Eckmann, C. R., S. L. Crittenden, N. Suh, and J. Kimble, 2004 GLD-3 and control of the mitosis/meiosis decision in the germline of Caenorhabditis elegans. Genetics 168: 147160. https://doi.org/10.1534/genetics.104.029264

Edelstein, A., N. Amodaj, K. Hoover, R. Vale, and N. Stuurman, 2010 Computer control of microscopes using $\mu$ Manager. Curr. Protoc. Mol. Biol. Chapter 14: Unit14.20.

Edelstein, A. D., M. A. Tsuchida, N. Amodaj, H. Pinkard, R. D. Vale et al., 2014 Advanced methods of microscope control using $\mu$ Manager software. J. Biol. Methods 1: 10.

Edgley, M. L., and D. L. Riddle, 2001 LG II balancer chromosomes in Caenorhabditis elegans: mT1(II;III) and the mIn1 set of dominantly and recessively marked inversions. Mol. Genet. Genomics 266: 385-395. https://doi.org/10.1007/s004380100523

Edgley, M. L., D. L. Baillie, D. L. Riddle, and A. M. Rose, 2006 Genetic balancers (April 6, 2006), WormBook, ed. The C. elegans Research Community WormBook, doi/10.1895/ wormbook.1.89.1, http://www.wormbook.org.

Félix, M. A., and A. Wagner, 2008 Robustness and evolution: concepts, insights and challenges from a developmental model system. Heredity (Edinb) 100: 132-140. https://doi.org/10.1038/ sj.hdy. 6800915

Finney, M., and G. Ruvkun, 1990 The unc-86 gene product couples cell lineage and cell identity in C. elegans. Cell 63: 895905. https://doi.org/10.1016/0092-8674(90)90493-X

Forbes, A., and R. Lehmann, 1998 Nanos and Pumilio have critical roles in the development and function of Drosophila germline stem cells. Development 125: 679-690.

Fraser, A. G., R. S. Kamath, P. Zipperlen, M. Martinez-Campos, M. Sohrmann et al., 2000 Functional genomic analysis of C. elegans chromosome I by systematic RNA interference. Nature 408: 325-330. https://doi.org/10.1038/35042517
Frøkjær-Jensen, C., M. W. Davis, M. Sarov, J. Taylor, S. Flibotte et al., 2014 Random and targeted transgene insertion in Caenorhabditis elegans using a modified Mos1 transposon. Nat. Methods 11: 529-534. https://doi.org/10.1038/nmeth.2889

Gibson, D. G., 2009 Synthesis of DNA fragments in yeast by onestep assembly of overlapping oligonucleotides. Nucleic Acids Res. 37: 6984-6990. https://doi.org/10.1093/nar/gkp687

Gibson, D. G., L. Young, R. Y. Chuang, J. C. Venter, C. A. Hutchison, III et al., 2009 Enzymatic assembly of DNA molecules up to several hundred kilobases. Nat. Methods 6: 343-345. https:// doi.org/10.1038/nmeth.1318

Gietz, R. D., and R. H. Schiestl, 2007 High-efficiency yeast transformation using the LiAc/SS carrier DNA/PEG method. Nat. Protoc. 2: 31-34. https://doi.org/10.1038/nprot.2007.13

Gross-Thebing, T., S. Yigit, J. Pfeiffer, M. Reichman-Fried, J. Bandemer et al., 2017 The vertebrate protein dead end maintains primordial germ cell fate by inhibiting somatic differentiation. Dev. Cell 43: 704-715.e5. https://doi.org/10.1016/j.devcel.2017.11.019

Hafner, M., M. Landthaler, L. Burger, M. Khorshid, J. Hausser et al., 2010 Transcriptome-wide identification of RNA-binding protein and microRNA target sites by PAR-CLIP. Cell 141: 129141. https://doi.org/10.1016/j.cell.2010.03.009

Haupt, K. A., A. L. Enright, A. S. Ferdous, A. M. Kershner, H. Shin et al., 2019 The molecular basis of LST-1 self-renewal activity and its control of stem cell pool size. Development 146: dev181644. https://doi.org/10.1242/dev.181644

Hodgkin, J., H. R. Horvitz, and S. Brenner, 1979 Nondisjunction mutants of the nematode Caenorhabditis elegans. Genetics 91: 67-94.

Hook, B., D. Bernstein, B. Zhang, and M. Wickens, 2005 RNAprotein interactions in the yeast three-hybrid system: affinity, sensitivity, and enhanced library screening. RNA 11: 227-233. https://doi.org/10.1261/rna.7202705

Hubstenberger, A., C. Cameron, R. Shtofman, S. Gutman, and T. C. Evans, 2012 A network of PUF proteins and Ras signaling promote mRNA repression and oogenesis in C. elegans. Dev. Biol. 366: 218-231. https://doi.org/10.1016/j.ydbio.2012.03.019

Hubstenberger, A., S. L. Noble, C. Cameron, and T. C. Evans, 2013 Translation repressors, an RNA helicase, and developmental cues control RNP phase transitions during early development. Dev. Cell 27: 161-173. https://doi.org/10.1016/ j.devcel.2013.09.024

Kadyk, L. C., and J. Kimble, 1998 Genetic regulation of entry into meiosis in Caenorhabditis elegans. Development 125: 18031813.

Kershner, A., S. L. Crittenden, K. Friend, E. B. Sorensen, D. F. Porter et al., 2013 Germline stem cells and their regulation in the nematode Caenorhabditis elegans. Adv. Exp. Med. Biol. 786: 29-46. https://doi.org/10.1007/978-94-007-6621-1 3

Kershner, A. M., and J. Kimble, 2010 Genome-wide analysis of mRNA targets for Caenorhabditis elegans FBF, a conserved stem cell regulator. Proc. Natl. Acad. Sci. USA 107: 3936-3941. https://doi.org/10.1073/pnas.1000495107

Kershner, A. M., H. Shin, T. J. Hansen, and J. Kimble, 2014 Discovery of two GLP-1/Notch target genes that account for the role of GLP-1/Notch signaling in stem cell maintenance. Proc. Natl. Acad. Sci. USA 111: 3739-3744. https://doi.org/ 10.1073/pnas.1401861111

Kimble, J., and S. L. Crittenden, 2005 Germline proliferation and its control (August 15, 2005), WormBook, ed. The C. elegans Research Community WormBook, doi/10.1895/wormbook.1.13.1, http:// www.wormbook.org.https://doi.org/10.1895/wormbook.1.13.1

Kimble, J., and S. L. Crittenden, 2007 Controls of germline stem cells, entry into meiosis, and the sperm/oocyte decision in Caenorhabditis elegans. Annu. Rev. Cell Dev. Biol. 23: 405-433. https://doi.org/10.1146/annurev.cellbio.23.090506.123326 
Kimble, J. E., and J. G. White, 1981 On the control of germ cell development in Caenorhabditis elegans. Dev. Biol. 81: 208-219. https://doi.org/10.1016/0012-1606(81)90284-0

Koh, Y. Y., L. Opperman, C. Stumpf, A. Mandan, S. Keles et al., 2009 A single C. elegans PUF protein binds RNA in multiple modes. RNA 15: 1090-1099. https://doi.org/10.1261/ rna.1545309

Lamont, L. B., S. L. Crittenden, D. Bernstein, M. Wickens, and J. Kimble, 2004 FBF-1 and FBF-2 regulate the size of the mitotic region in the C. elegans germline. Dev. Cell 7: 697-707. https:// doi.org/10.1016/j.devcel.2004.09.013

Lee, C., E. B. Sorensen, T. R. Lynch, and J. Kimble, 2016 C. elegans GLP-1/Notch activates transcription in a probability gradient across the germline stem cell pool. eLife 5: e18370. https:// doi.org/10.7554/eLife.18370

Lin, H., and A. C. Spradling, 1997 A novel group of pumilio mutations affects the asymmetric division of germline stem cells in the Drosophila ovary. Development 124: 2463-2476.

Liu, Q., C. Stumpf, C. Thomas, M. Wickens, and E. S. Haag, 2012 Context-dependent function of a conserved translational regulatory module. Development 139: 1509-1521. https:// doi.org/10.1242/dev.070128

Merritt, C., and G. Seydoux, 2010 The Puf RNA-binding proteins FBF-1 and FBF-2 inhibit the expression of synaptonemal complex proteins in germline stem cells. Development 137: 17871798. https://doi.org/10.1242/dev.050799

Merritt, C., D. Rasoloson, D. Ko, and G. Seydoux, 2008 3' UTRs are the primary regulators of gene expression in the C. elegans germline. Curr. Biol. 18: 1476-1482. https://doi.org/10.1016/ j.cub.2008.08.013

Miller, D. M., and D. C. Shakes, 1995 Immunofluorescence microscopy, pp. 365-394 in Caenorhabditis elegans: Modern Biological Analysis of an Organism, edited by H. F. Epstein and D. C. Shakes. Academic Press, Inc., San Diego. https://doi.org/ 10.1016/S0091-679X(08)61396-5

Naudin, C., A. Hattabi, F. Michelet, A. Miri-Nezhad, A. Benyoucef et al., 2017 PUMILIO/FOXP1 signaling drives expansion of hematopoietic stem/progenitor and leukemia cells. Blood 129: 2493-2506. https://doi.org/10.1182/blood-2016-10-747436

Paix, A., A. Folkmann, D. Rasoloson, and G. Seydoux, 2015 High efficiency, homology-directed genome editing in Caenorhabditis elegans using CRISPR-Cas9 ribonucleoprotein complexes. Genetics 201: 47-54. https://doi.org/10.1534/genetics.115.179382

Porter, D. F., A. Prasad, B. H. Carrick, P. Kroll-Connor, M. Wickens et al., 2019 Toward identifying subnetworks from FBF binding landscapes in Caenorhabditis spermatogenic or oogenic germlines. G3 (Bethesda) 9: 153-165. https://doi.org/10.1534/ g3.118.200300

Prasad, A., D. F. Porter, P. L. Kroll-Conner, I. Mohanty, A. R. Ryan et al., 2016 The PUF binding landscape in metazoan germ cells. RNA 22: 1026-1043. https://doi.org/10.1261/rna.055871.116

Preibisch, S., S. Saalfeld, and P. Tomancak, 2009 Globally optimal stitching of tiled 3D microscopic image acquisitions. Bioinformatics 25: 1463-1465. https://doi.org/10.1093/bioinformatics/btp184

Qiu, C., V. D. Bhat, S. Rajeev, C. Zhang, A. E. Lasley et al., 2019 A crystal structure of a collaborative RNA regulatory complex reveals mechanisms to refine target specificity. eLife 8: e48968. https://doi.org/10.7554/eLife.48968

Salvetti, A., L. Rossi, A. Lena, R. Batistoni, P. Deri et al., 2005 DjPum, a homologue of Drosophila Pumilio, is essential to planarian stem cell maintenance. Development 132: 18631874. https://doi.org/10.1242/dev.01785

Schindelin, J., I. Arganda-Carreras, E. Frise, V. Kaynig, M. Longair et al., 2012 Fiji: an open-source platform for biological-image analysis. Nat. Methods 9: 676-682. https://doi.org/10.1038/ nmeth.2019
Seidel, H. S., and J. Kimble, 2011 The oogenic germline starvation response in C. elegans. PLoS One 6: e28074. https:// doi.org/10.1371/journal.pone.0028074

Seidel, H. S., and J. Kimble, 2015 Cell-cycle quiescence maintains Caenorhabditis elegans germline stem cells independent of GLP1/Notch. eLife 4: e10832. https://doi.org/10.7554/eLife.10832

Shin, H., K. A. Haupt, A. M. Kershner, P. Kroll-Conner, M. Wickens et al., 2017 SYGL-1 and LST-1 link niche signaling to PUF RNA repression for stem cell maintenance in Caenorhabditis elegans. PLoS Genet. 13: e1007121. https://doi.org/10.1371/journal.pgen. 1007121

Siegfried, K., and J. Kimble, 2002 POP-1 controls axis formation during early gonadogenesis in C. elegans. Development 129: 443-453.

Slaidina, M., and R. Lehmann, 2014 Translational control in germline stem cell development. J. Cell Biol. 207: 13-21. https://doi.org/10.1083/jcb.201407102

Thompson, B. E., D. S. Bernstein, J. L. Bachorik, A. G. Petcherski, M. Wickens et al., 2005 Dose-dependent control of proliferation and sperm specification by FOG-1/CPEB. Development 132: 3471-3481. https://doi.org/10.1242/dev.01921

Thompson, O., M. Edgley, P. Strasbourger, S. Flibotte, B. Ewing et al., 2013 The million mutation project: a new approach to genetics in Caenorhabditis elegans. Genome Res. 23: 1749-1762. https://doi.org/10.1101/gr.157651.113

Timmons, L., and A. Fire, 1998 Specific interference by ingested dsRNA. Nature 395: 854. https://doi.org/10.1038/27579

Tsukamoto, T., M. D. Gearhart, C. A. Spike, G. Huelgas-Morales, M. Mews et al., 2017 LIN-41 and OMA ribonucleoprotein complexes mediate a translational repression-to-activation switch controlling oocyte meiotic maturation and the oocyte-to-embryo transition in Caenorhabditis elegans. Genetics 206: 2007-2039. https://doi.org/10.1534/genetics.117.203174

Voronina, E., 2013 The diverse functions of germline P-granules in Caenorhabditis elegans. Mol. Reprod. Dev. 80: 624-631. https://doi.org/10.1002/mrd.22136

Voronina, E., A. Paix, and G. Seydoux, 2012 The P granule component PGL-1 promotes the localization and silencing activity of the PUF protein FBF-2 in germline stem cells. Development 139: 3732-3740. https://doi.org/10.1242/dev.083980

Wang, X., J. R. Olson, D. Rasoloson, M. Ellenbecker, J. Bailey et al., 2016 Dynein light chain DLC-1 promotes localization and function of the PUF protein FBF-2 in germline progenitor cells. Development 143: 4643-4653. https://doi.org/10.1242/ dev.140921

Wang, Y., L. Opperman, M. Wickens, and T. M. Hall, 2009 Structural basis for specific recognition of multiple mRNA targets by a PUF regulatory protein. Proc. Natl. Acad. Sci. USA 106: 20186-20191. https://doi.org/10.1073/ pnas.0812076106

Wickens, M., D. S. Bernstein, J. Kimble, and R. Parker, 2002 A PUF family portrait: 3'UTR regulation as a way of life. Trends Genet. 18: 150-157. https://doi.org/10.1016/S01689525(01)02616-6

Yamaji, M., M. Jishage, C. Meyer, H. Suryawanshi, E. Der et al., 2017 DND1 maintains germline stem cells via recruitment of the CCR4-NOT complex to target mRNAs. Nature 543: 568572. https://doi.org/10.1038/nature21690

Ye, J., and R. Blelloch, 2014 Regulation of pluripotency by RNA binding proteins. Cell Stem Cell 15: 271-280. https://doi.org/ 10.1016/j.stem.2014.08.010

Zhang, M., D. Chen, J. Xia, W. Han, X. Cui et al., 2017 Posttranscriptional regulation of mouse neurogenesis by Pumilio proteins. Genes Dev. 31: 1354-1369. https://doi.org/10.1101/ gad.298752.117

Communicating editor: D. Greenstein 